ARTICLE

Received 29 Jun 2015 | Accepted 9 Feb 2016 | Published 23 Mar 2016

DOI: $10.1038 /$ ncomms11017

OPEN

\title{
VEGFR2 pY949 signalling regulates adherens junction integrity and metastatic spread
}

Xiujuan Li ${ }^{1, \star}$, Narendra Padhan ${ }^{1, \star}$, Elisabet O. Sjöström ${ }^{1}$ Francis P. Roche ${ }^{1}$, Chiara Testini ${ }^{1}$, Naoki Honkura ${ }^{1}$ Miguel Sáinz-Jaspeado1, Emma Gordon', Katie Bentley ${ }^{1,2}$, Andrew Philippides ${ }^{3}$, Vladimir Tolmachev', Elisabetta Dejana ${ }^{1,4}$, Radu V. Stan ${ }^{5}$, Dietmar Vestweber ${ }^{6}$, Kurt Ballmer-Hofer ${ }^{7}$, Christer Betsholtz ${ }^{1,8}$, Kristian Pietras ${ }^{9}$, Leif Jansson ${ }^{10} \&$ Lena Claesson-Welsh ${ }^{1}$

The specific role of VEGFA-induced permeability and vascular leakage in physiology and pathology has remained unclear. Here we show that VEGFA-induced vascular leakage depends on signalling initiated via the VEGFR2 phosphosite Y949, regulating dynamic c-Src and VE-cadherin phosphorylation. Abolished Y949 signalling in the mouse mutant Vegfr2 ${ }^{Y 949 F / Y 949 F}$ leads to VEGFA-resistant endothelial adherens junctions and a block in molecular extravasation. Vessels in Vegfr2 ${ }^{\text {Y } 949 F / Y 949 F}$ mice remain sensitive to inflammatory cytokines, and vascular morphology, blood pressure and flow parameters are normal.

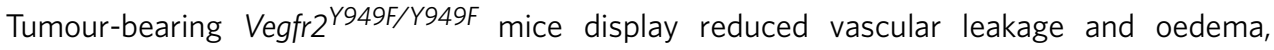
improved response to chemotherapy and, importantly, reduced metastatic spread. The inflammatory infiltration in the tumour micro-environment is unaffected. Blocking VEGFAinduced disassembly of endothelial junctions, thereby suppressing tumour oedema and metastatic spread, may be preferable to full vascular suppression in the treatment of certain cancer forms.

\footnotetext{
${ }^{1}$ Department of Immunology, Genetics and Pathology, Rudbeck Laboratory, Science for Life Laboratory, Uppsala University, 75185 Uppsala, Sweden.

${ }^{2}$ Beth Israel Deaconess Medical Center, Harvard Medical School, 330 Brookline Avenue, Boston, Massachusetts 02215, USA. ${ }^{3}$ Centre for Computational Neuroscience and Robotics, University of Sussex, Chichester $1 \mathrm{Cl}$ 104, Brighton BN1 9RH, UK. ${ }^{4}$ C/O IFOM-IEO Campus, Via Adamello, 16, 20139 Milan, Italy. ${ }^{5}$ Department of Pathology, Dartmouth College, Geisel School of Medicine at Dartmouth, Lebanon, New Hampshire 03756, USA. ${ }^{6}$ Department of Vascular Cell Biology, Max Planck Institute for Molecular Biomedicine, Röntgenstraße 20, 48149 Münster, Germany. ${ }^{7}$ Biomolecular Research, Molecular Cell Biology, Paul-Scherrer Institute, 5232 Villigen-PSI, Switzerland. ${ }^{8}$ Karolinska Institutet, Dept. Medical Biochemistry and Biophysics, Div. Vascular Biology, 17177 Stockholm, Sweden. ${ }^{9}$ Translational Cancer Research, Medicon Village, Lund University, Building 404:A3, 22381 Lund, Sweden. ${ }^{10}$ Department of Medical Cell Biology, Biomedical Center, Uppsala University, Box 571, 75123 Uppsala, Sweden. ${ }^{\star}$ These authors contributed equally to this work. Correspondence and requests for materials should be addressed to L.C-W. (email: lena.welsh@igp.uu.se).
} 
C ancer is characterized by a dysfunctional and morphologically abnormal vasculature with elevated permeability and sparse pericyte and basement membrane coating, resulting in unstable vessels and poor blood flow ${ }^{1}$. The dismal condition of the tumour vasculature is attributed to the hypoxic tumour micro-environment and the accompanying inflammation resulting in the production of a broad range of growth modulatory factors including vascular endothelial growth factors (VEGFs), in particular VEGFA (ref. 2). VEGFA causes increased vascular leakage by disruption of adherens junctions created through homophilic interactions between vascular endothelial (VE)-cadherin molecules expressed on adjacent endothelial cells ${ }^{3}$. Vessel normalization by titrating an appropriate level of anti-VEGFA therapy improves the efficacy of irradiation and chemotherapy ${ }^{4}$. However, sustained antiVEGFA therapy deteriorates vessel function and may cause increased hypoxia, increased local tumour cell invasion and increased metastatic propensity (for a review, see ref. 5). The role of VEGFA-induced permeability compared with VEGFAregulated migration, survival and proliferation of endothelial cells is unknown. Moreover, the signal transduction pathways induced by VEGFA to promote increased vascular permeability have remained unidentified.

VEGFA exerts its effects via two endothelial cell receptor tyrosine kinases denoted VEGFR1 and VEGFR2 (ref. 6). VEGFR1 binds and neutralizes VEGFA, thereby exerting a negative regulatory effect on endothelial cells, while VEGFR2 is essential in all known VEGFA biology ${ }^{6}$. VEGFR2 becomes activated and phosphorylated on tyrosine residues in response to VEGFA: Y949, Y1052, Y1057, Y1173 and Y1212 (mouse sequence numbers) ${ }^{7}$. The Y949 phosphosite in VEGFR2 (Y951 in human VEGFR2) presents a specific binding site for the $\mathrm{T}$ cell-specific adaptor (TSAd). TSAd is implicated in VEGFA-induced permeability, by regulating VEGFR2-dependent c-Src signalling at endothelial cell junctions ${ }^{8}$. The Y1052/1057 residues, located on the tyrosine kinase activation loop, are required for full kinase activity (see ref. 6 and references therein). The phosphorylated Y1173 binds phospholipase $\mathrm{C} \gamma$, of importance for endothelial ERK1/2 pathway activation ${ }^{9}$. A phenylalanine knock-in mouse Vegfr $2^{Y 1773 F / Y 1173 F}$ is embryonically lethal due to arrested endothelial cell development ${ }^{10}$. The Vegfr2 $2^{Y 1212 F / Y 1212 F}$ mouse lacks a developmental phenotype on a mixed background ${ }^{10}$.

The aim of the current study was to examine the consequence of specific suppression of VEGFA-induced permeability on cancer progression. VEGFA-induced molecular extravasation was attenuated in the Vegfr2 ${ }^{Y 949 F / Y 949 F}$ mouse due to perturbed signalling through the TSAd/c-Src/VE-cadherin pathway resulting in VEGFA-resistant adherens junctions. The Y949F mutation however did not perturb other aspects of VEGFA-regulated vessel biology. The loss of VEGFA-regulated leakage was compatible with vascular development and a morphologically normal vasculature including the presence of fenestrated endothelium in adult organs. Blood flow and blood pressure changes in response to VEGFA were unaffected by the mutation. In cancer, the loss of VEGFA-regulated leakage was manifested as reduced tumour oedema, improved responsiveness to chemotherapy and suppressed metastatic spread due to an arrest in tumour cell intravasation.

\section{Results}

Vegfr $2^{Y 949 F / Y 949 F}$ vessels do not leak in response to VEGFA. Vegfr $2^{\text {Y949F/Y949F }}$ knock-in mice were created using Velocigene technology ${ }^{11}$ and used in this study after selection cassette removal, sequence verification and extensive backcrossing onto the $\mathrm{C} 57 \mathrm{Bl} / 6$ background (Supplementary Fig. 1). The mutant mice were phenotypically normal and expressed similar levels of VEGFR2 protein in the vasculature as wild-type (WT) mice (see below). To determine the consequence of Vegfr $2^{Y 949 F / Y 949 F}$ mutation on VEGFA-induced vascular leakage in vivo, we employed two well-established assays, microsphere extravasation in the tracheal vasculature and Miles extravasation assay in the dermis. To induce microsphere extravasation, VEGFA or vehicle was administered by tail vein injection together with fluorescent 30-nm microspheres. VEGFA-induced extravasation of microspheres, lodged beneath the basement membrane in postcapillary venules, was significantly reduced at 60 min of circulation in the Vegfr $2^{Y 949 F / Y 949 F}$ trachea compared with WT (Fig. 1a,b). In contrast, tail vein-administered histamine induced similar levels of microsphere extravasation in WT and Vegfr $2^{Y 949 F / Y 949 F}$ trachea venules (Fig. 1c,d). Moreover, VEGFA induced a significant increase of Evans' blue leakage in the skin (Miles assay) in the WT but not in the Vegfr $2^{\text {Y949F/Y949F }}$ mouse (Fig. 1e).

We have previously shown that the phosphorylated Y949 site in VEGFR2 serves as a binding site for Src homology 2 (SH2) domain of $\mathrm{TSAd}^{8}$, which in turn binds the $\mathrm{SH} 3$ domain of c-Src. $\mathrm{c}-\mathrm{Src}$ is known to regulate VE-cadherin and adherens junctions integrity ${ }^{12}$. We verified VEGFA-induced VEGFR2/TSAd complex formation in the WT and lack of TSAd binding to mutant

Figure 1 | Arrest in VEGFA-induced vascular leakage in Vegfr2 ${ }^{\text {Y949F/Y949F }}$ mice. (a) Tracheal vessel leakage in WT (left) and Vegfr2 ${ }^{\text {Y949F/Y949F }}$ (Y949F; right) mice after tail vein injection of VEGFA or PBS combined with $30 \mathrm{~nm}$ fluorescent microspheres (green). Arrows: microsphere leakage from CD31+ vessels (red). Scale bar, $50 \mu \mathrm{m}$. (b) Microsphere area per CD31-positive area fraction in VEGFA/PBS samples from a. $n=3$ mice per genotype. Two-way ANOVA: $P$ (treatment $)<0.0001 ; P$ (genotype $)=0.001 ; P$ (interaction $)=0.0008$. (c) Tracheal vessel leakage in response to tail vein-injected histamine (as in a), in WT and Vegfr2 ${ }^{Y 949 F / Y 949 F}$ (Y949F) mice. (d) Microsphere area per CD31-positive area fraction in histamine/PBS samples from c. $n=3-6$ mice per genotype. Two-way ANOVA: $P$ (treatment) $<0.0001 ; P($ genotype $)=0.84 ; P$ (interaction) $=0.80$. (e) Evans' blue extravasation induced by VEGFA/PBS in the dermis of WT and Vegfr2 ${ }^{Y 949 F / Y 949 F}$ (Y949F) mice. Values were normalized to tissue weight. $n=3$ mice per genotype. Two-way ANOVA: $P$ (treatment $)=0.0053 ; P$ (genotype $)=0.0206 ; P$ (interaction $)=0.0482$. (f) VEGFR2/TSAd complex formation in isolated mouse lung endothelial cells


VEGFR2. Total lysates were immunoblotted for TSAd and tubulin. Positive control, total lysate. Molecular weight markers to the right. (g) VE-cadherin immunostaining (yellow-black heatmap) on trachea from WT and Vegfr2 Y949F/Y949F (Y949F) mice, tail vein-injected with VEGFA/PBS and microspheres (pink-red heatmap). Images were rendered using structured illumination microscopy. Note VE-cadherin rearrangement in VEGFA-injected WT trachea. Scale bar, $10 \mu \mathrm{m}$. (h) VE-cadherin fragments per $\mu \mathrm{m}$ vessel length from $\mathbf{g}$. $n=3-6$ individual tissues per genotype. Total vessel length assessed: WT/PBS, $236 \mu \mathrm{m} ;$ WT/VEGFA, $308 \mu \mathrm{m}$; Y949F/PBS, $150 \mu \mathrm{m}$; and Y949F/VEGFA, $145 \mu \mathrm{m}$. Two-way ANOVA: $P$ (treatment) $=0.0022 ; P(\mathrm{genotype})=0.0065$; $P$ (interaction) $=0.0164$. (i) VE-cadherin immunostaining (red) on endothelial cells isolated from WT and Vegfr2 ${ }^{\text {Y949F/Y949F }}$ (Y949F) mouse lungs and then treated or not with VEGFA (100 $\mathrm{ng} \mathrm{ml}^{-1}$ ). Hoechst 33342 (blue) staining shows nuclei. Scale bar, $20 \mu \mathrm{m}$. (j) VE-cadherin-positive staining per cell; samples generated as in $i . n=30$ cells per condition. Two-way ANOVA: $P$ (treatment) $=0.0189 ; P$ (genotype $)=0.099 ; P($ interaction) $=0.0741$. Data presented as mean \pm s.e.m. Two-way ANOVA with Tukey's post hoc test, ${ }^{\star} P<0.05,{ }^{\star \star} P<0.01,{ }^{\star \star \star} P<0.001,{ }^{\star \star \star \star} P<0.0001$. Experiments were performed at least three independent times. 
VEGFR2-Y949F, by immunoblotting on isolated endothelial cells



We next analysed the consequence of the VEGFR2-Y949F mutation of VE-cadherin stability. Immunostaining for VEcadherin visualised continuous cell borders in WT and Vegfr $2^{\text {Y949F/Y949F }}$ tracheal vessels, analysed using structured illumination microscopy imaging (Fig. 1g). In response to
VEGFA, the WT tracheal vessels, but not Vegfr2 ${ }^{Y 949 F / Y 949 F}$ vessels, showed a significant increase in fragmented VE-cadherin staining (Fig. 1g, quantified in $\mathrm{h}$ ) indicative of VE-cadherin internalization and breakdown of the adherens junctions causing transient vascular leakage ${ }^{13}$. The difference in VE-cadherin dynamics between the genotypes was validated using endothelial cells isolated from mouse lungs. VEGFA promoted a

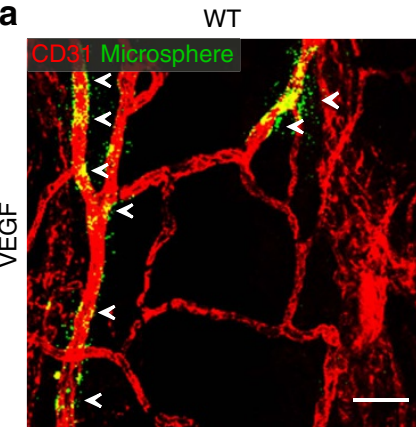

C
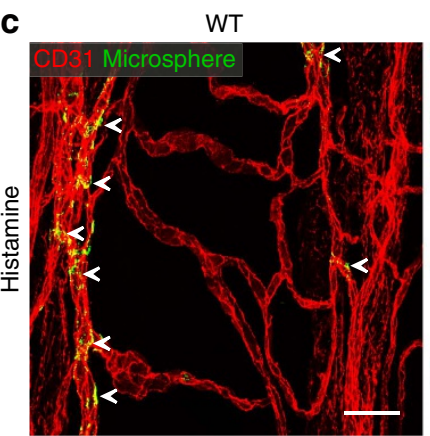

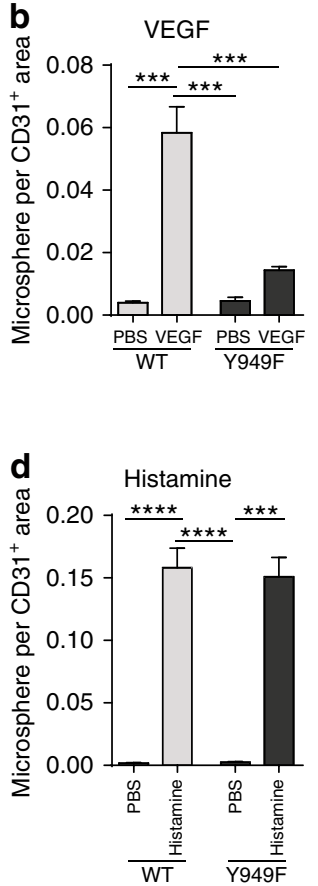

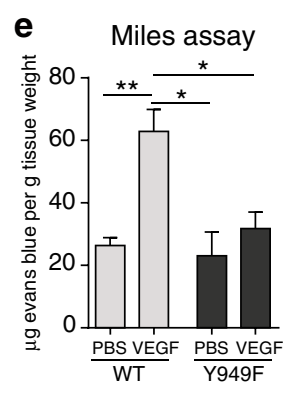

f

IP:VEGFR2 from isolated endothelial cells from mouse lungs
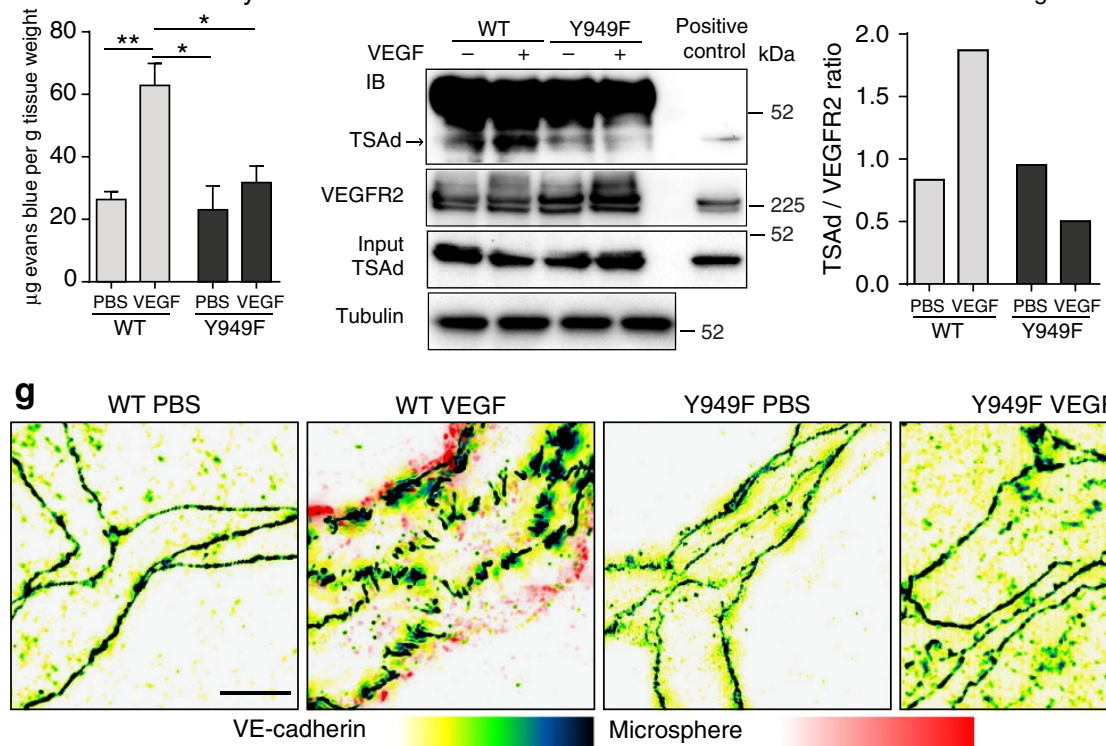

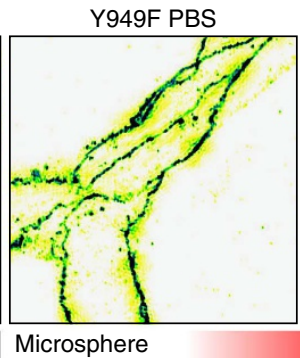

Y949F PBS

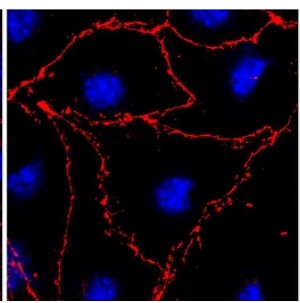

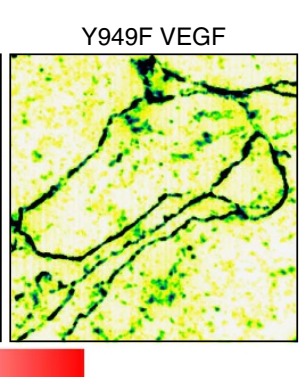

Y949F VEGF

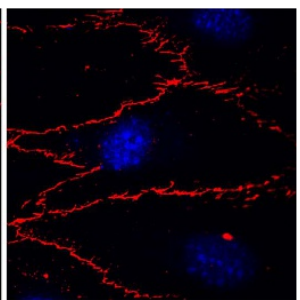

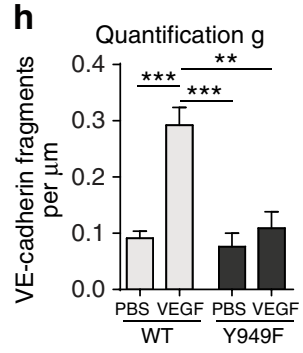

j




VE-cadherin junction disintegration in the WT cells but not in the Vegfr2 ${ }^{\text {Y949F/Y949F }}$ cells, resulting in a significantly increased VE-cadherin-positive staining area in the VEGFA-treated WT cells (Fig. 1i,j).

Vegfr $2^{\text {Y949F/Y949F }}$ vessel characteristics. The Vegfr2 $2^{Y 949 F / Y 949 F}$ mice showed no developmental vascular abnormality, for example, in the embryonic day 11.5 (E11.5) hindbrain (Supplementary Fig. 2a-d) and were born at the expected Mendelian ratios. There was also no apparent effect of the mutation on the development and organization of lymphatics (see Supplementary Fig. 2e for tracheal lymphatics). The postnatal retinal vasculature develops in a strictly regulated, VEGFA-dependent manner to establish the junctional barrier ${ }^{14}$. a



e

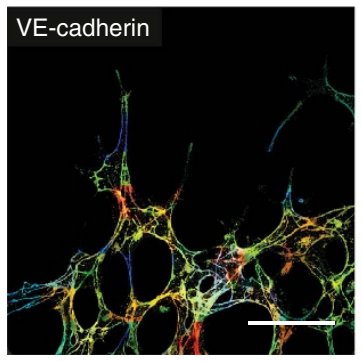

h WT kidney glomerulus

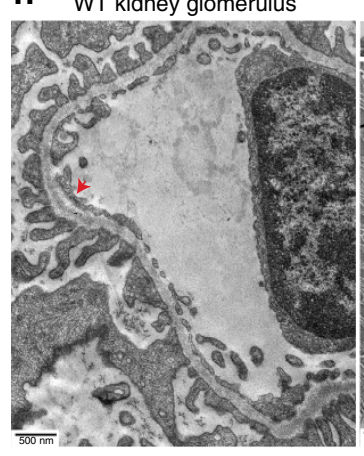

$\mathbf{k}$

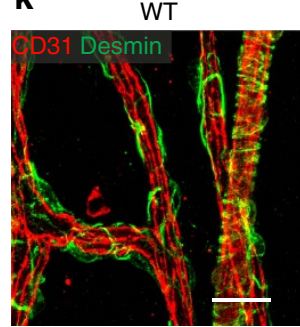

m WT

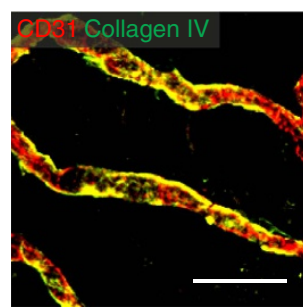

Y949F
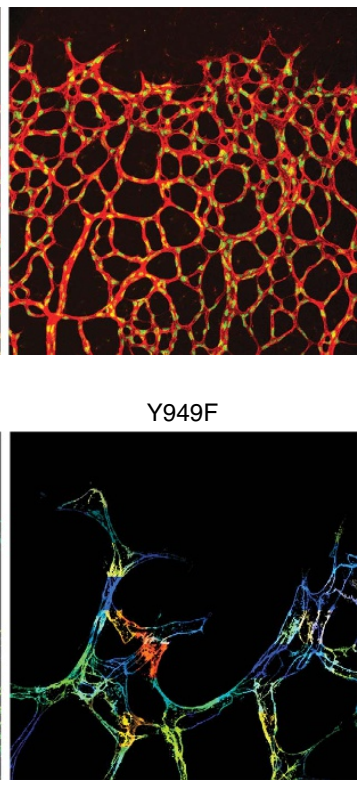

WT peritubular cap

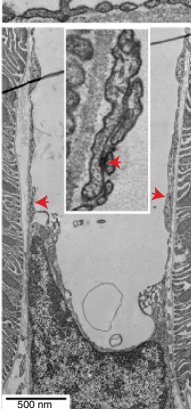

Y949F

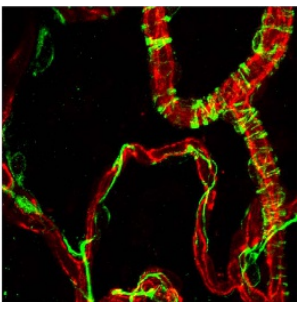

Y949F

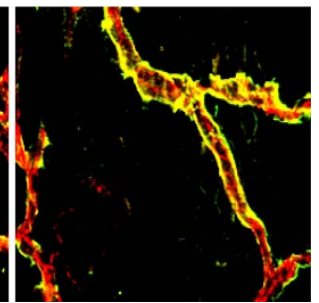

b

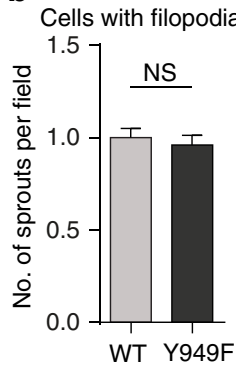

C

d


$\mathbf{f}$
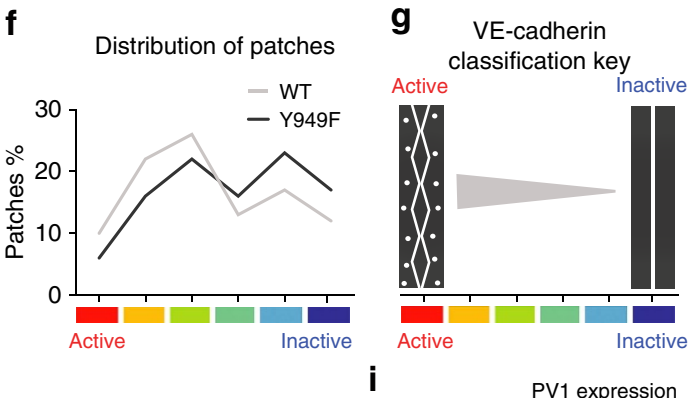

P1 V1




n Basement membrane

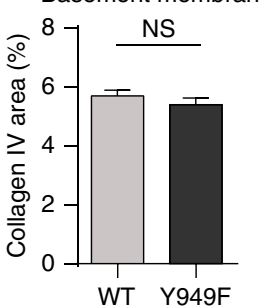

$\mathbf{0}$

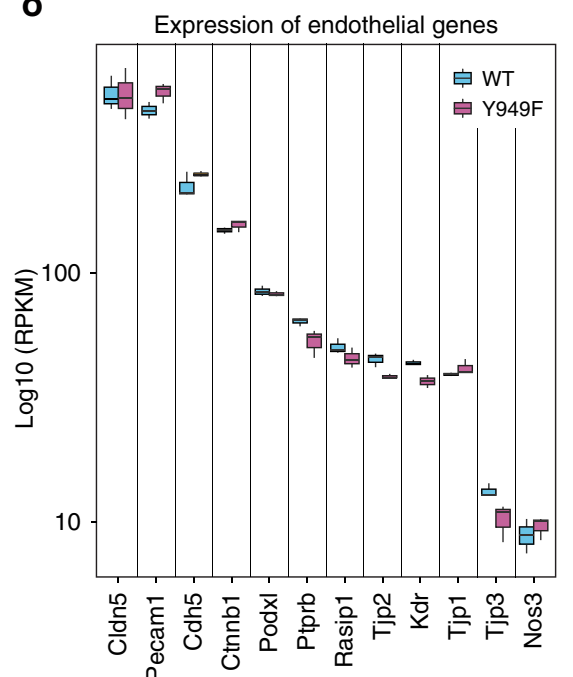


There was no difference in retinal vascular development between

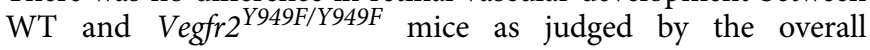
morphology of the vascular network, the abundance of endothelial tip cells and filopodia, and the total vascular area (Fig. 2a-d). Thus, development of the retinal vasculature does not depend on VEGFR2-Y949 signalling. However, the morphology of the VE-cadherin immunostaining differed between the two genotypes. The VE-cadherin pattern at individual junctions was evaluated using established image analysis software ${ }^{15}$, classifying junctions in a graded scale from 'active' - that is, being irregular/ serrated with vesicular/diffuse appearance-to 'inactive' ones, presenting a straighter and less vesicular morphology (Fig. 2e, see Fig. 2f,g for quantification and schematic outline). By this criterion, the WT retinal vasculature displayed a significantly higher prevalence of active adherens junctions compared with the Vegfr $2^{\text {Y } 949 F / Y 949 F}$ retinal vasculature (Fig. 2f; active junction patches was $58.9 \%$ (WT) versus $48.1 \%$ (Vegfr $\left.2^{Y 949 F / Y 949 F}\right)$;

\begin{tabular}{|c|c|c|c|}
\hline Strain & $\begin{array}{c}\text { WT } \\
(n=9)\end{array}$ & $\begin{array}{l}\text { Y949F } \\
(n=8)\end{array}$ & $\begin{array}{c}P \\
\text { value }\end{array}$ \\
\hline $\mathrm{pH}$ & $7.29 \pm 0.01$ & $7.32 \pm 0.02$ & 0.288 \\
\hline Arterial $\mathrm{pCO}_{2}(\mathrm{kPa})$ & $5.8 \pm 0.3$ & $5.5 \pm 0.3$ & 0.596 \\
\hline Arterial $\mathrm{pO}_{2}(\mathrm{kPa})$ & $13.1 \pm 0.9$ & $14.7 \pm 0.6$ & 0.167 \\
\hline Base excess $\left(\mathrm{mmoll}^{-1}\right)$ & $-5.8 \pm 0.6$ & $-4.6 \pm 1.0$ & 0.341 \\
\hline Arterial $\mathrm{HCO}_{3}\left(\mathrm{mmoll}^{-1}\right)$ & $20.7 \pm 0.7$ & $21.3 \pm 1.1$ & 0.661 \\
\hline Arterial oxygen saturation (\%) & $95.6 \pm 1.0$ & $97.5 \pm 0.4$ & 0.185 \\
\hline $\mathrm{S}-\mathrm{Na}^{+}(\mathrm{mmoll}-1)$ & $144.8 \pm 0.6$ & $143.2 \pm 0.5$ & 0.09 \\
\hline $\mathrm{S}-\mathrm{K}^{+}(\mathrm{mmoll}-1)$ & $5.1 \pm 0.2$ & $5.2 \pm 0.2$ & 0.757 \\
\hline Haematocrit (\%) & $32.3 \pm 1.1$ & $33.0 \pm 1.6$ & 0.73 \\
\hline Haemoglobin $\left(\left.g\right|^{-1}\right)$ & $110 \pm 4$ & $112 \pm 5$ & 0.732 \\
\hline \multicolumn{4}{|l|}{ Blood flow values* } \\
\hline \multicolumn{4}{|l|}{ Pancreatic } \\
\hline Whole gland & $1.76 \pm 0.36$ & $1.42 \pm 0.22$ & 0.458 \\
\hline Caput & $2.10 \pm 0.48$ & $1.47 \pm 0.20$ & 0.532 \\
\hline Cauda & $1.52 \pm 0.32$ & $1.38 \pm 0.24$ & 0.735 \\
\hline Duodenal & $4.57 \pm 1.21$ & $3.60 \pm 0.70$ & 0.514 \\
\hline Colonic & $1.51 \pm 0.24$ & $1.48 \pm 0.47$ & 0.361 \\
\hline Lung & $0.15 \pm 0.07$ & $0.21 \pm 0.09$ & 0.665 \\
\hline Kidney & $7.15 \pm 1.65$ & $5.69 \pm 1.28$ & 0.503 \\
\hline CNS & $1.07 \pm 0.18$ & $1.64 \pm 0.48$ & 0.47 \\
\hline Fat & $0.013 \pm 0.004$ & $0.008 \pm 0.003$ & 0.768 \\
\hline
\end{tabular}

CNS, central nervous system

Values are means \pm s.e.m

${ }^{*} \mathrm{ml} \mathrm{min}{ }^{-1} \times \mathrm{g}$ organ weight.
$P=0.0367$, calculated using median\% in one-tailed Wilcoxon rank sum test.

Still, the enhanced barrier morphology in the VEGFR2-Y949F mutant did not interfere with overall vascular morphology or physiology. The adult vascular ultramorphology of the kidney glomeruli and peritubular capillaries (Fig. $2 \mathrm{~h}$ ) and the pancreas (Supplementary Fig. 2f) was normal in the Vegfr2 ${ }^{Y 949 F / Y 949 F}$ mouse. Moreover, expression of plasmalemma vesicle-associated protein-1 (PV1), which is required for the formation of diaphragms covering endothelial fenestrae in vessels of endocrine organs $^{16}$ was not affected by the VEGFR2-Y949F mutation (Fig. 2i).

To exclude the possibility that vascular leakage arrest in the Vegfr $2^{\text {Y949F/Y949F }}$ mouse was influenced by blood pressure/flow parameters, a series of in vivo tests were performed. The resting blood pressure in the descending aorta was monitored continuously while infusing VEGFA or phosphate-buffered saline (PBS) by tail vein injection. VEGFA, but not PBS, caused a significant drop in blood pressure as an indication of vessel dilation, in both WT and Vegfr2 $2^{Y 949 F / Y 949 F}$ mice (Fig. 2j). Moreover, blood parameters including arterial gases, oxygen saturation, serum $\mathrm{Na}^{+} / \mathrm{K}^{+}$levels, as well as the haematocrit/ haemoglobin levels were similar in the WT and Y949F conditions (Table 1). Tail vein injection of lectin showed that the WT and Vegfr $2^{Y 949 F / Y 949 F}$ tracheal vessels were perfused to the same extent (Supplementary Fig. 2g), supporting the notion that the leakage arrest in the mutant mouse vasculature was not due to altered circulation. Pericyte morphology (Fig. 2k,l) and basement membrane coating (Fig. 2m,n) were similar between the genotypes. Moreover, RNAseq analyses did not reveal major differences in expression levels of key endothelial transcripts between the WT and the Vegfr2 $2^{Y 949 F / Y 949 F}$ condition (Fig. 2o).

Reduced Vegfr2 ${ }^{\mathrm{Y} 949 \mathrm{~F} / \mathrm{Y} 949 \mathrm{~F}}$ glioblastoma vessel leakage. The integrity of the central nervous system vasculature is tightly controlled by the blood-brain barrier, in which endothelial junctions are particularly tight ${ }^{17}$. We asked whether VEGFAregulated vascular leakage has an impact on the growth and vascular permeability of brain tumours. The GL261 glioma model has many features reminiscent of human glioma, including abnormal vasculature, high VEGFA production and oedema ${ }^{18}$. GL261 glioma cells were injected in the right medial striatum of WT and Vegfr2 ${ }^{Y 949 F / Y 949 F}$ mice. There was no effect on tumour growth at any examined time point after cell inoculation (day 7 (D7), D14, D18 and D21; Fig. 3a shows tumour volume at D18). There was also no difference in the extent of perfused vessel

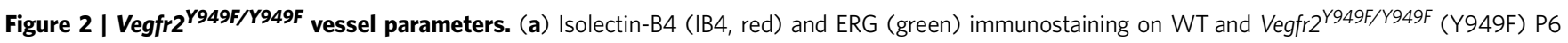
retinal vasculature. Scale bar, $100 \mu \mathrm{m}$. (b) Tip cells numbers. $n=3-4$ retinas per genotype. (c) IB4-positive vessel area normalized to WT. $n=3-4$ retinas per genotype. (d) Radial vessel outgrowth normalized to WT. $n=4-5$ retinas per genotype. (e) Heatmap of VE-cadherin morphology in WT and Vegfr2Y949F/Y949F (Y949F) P6 retinas. Scale bar, $50 \mu \mathrm{m}$. (f) Retinal images in e processed for VE-cadherin morphology, lying within the vascular mask, into 'patches' of $100 \times 100$ pixels ( $\times 63, n=6$ per group). The VE-cadherin morphology in each patch was hand-classified according to the classification key in $\mathbf{g}$. (g) Adherens junctions classification from 'active' (red; irregular/serrated morphology with diffuse/vesicular regions) to 'inactive' (blue; straighter morphology with less vesicular staining). (h) Transmission electron microscopy analysis of kidney glomerulus and peritubular capillary (cap) show normal appearance of junctions (red arrows) and fenestrae (insets) in the two genotypes. (i) PV1 protein immunoblot in lung lysates after injection of PBS (P) or VEGFA (V) and circulation for 1-10 min (P1; injection of PBS and $1 \mathrm{~min}$ circulation and so on). GAPDH; equal loading. Molecular weight markers to the right. (j). Blood pressure 10 min after saline or VEGFA infusion, monitored in the ascending aorta. Values (positive or negative) show diastolic blood pressure (BP) normalized to BP before VEGFA/PBS injection ( $70 \mathrm{~mm} \mathrm{Hg}$; NS between genotypes). $n=6-8$ mice per genotype. Student's $t$-test, ${ }^{\star} P<0.05$. Two-way ANOVA: $P($ treatment $)=0.0001 ; P($ genotype $)=0.738 ; P($ interaction $)=0.5321$. $(\mathbf{k})$ CD31-positive (red) tracheal endothelium and desmin-positive (green) pericytes. Scale bar; $50 \mu \mathrm{m}$. (I) Pericyte area per total area from $\mathbf{k} . n=4$ mice per genotype. (m) CD31-positive (red) tracheal endothelium and collagen IV-positive (green) basement membrane. Scale bar; $50 \mu \mathrm{m}$. (n) Collagen IV area per total area in $\mathbf{m}$. $n=3$ mice per genotype. (o) RNAseq analysis of total lung polyA + RNA. Data for selected endothelial genes as log10-scaled fold-change based on the frequency of normalized expression. For Vegfr2 (KDR) the Y949F mutant showed a - 0.24 (DESeq2log2) fold decrease compared with WT. Data presented as mean \pm s.e.m. Student's t-test (b-d) and Wilcoxon rank test (f); NS, not significant. Experiments were performed three independent times or performed once with at least three independent data sets $(\mathbf{j}, \mathbf{0})$. 
density (Fig. 3b). However, leakage of tail vein-injected cadaverine-Alexa-555, a small tracer that normally does not cross the blood-brain barrier ${ }^{19}$, was reduced in the

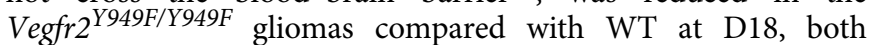
intratumourally and in the tumour periphery $(0-500 \mu \mathrm{m}$ peritumourally; Fig. 3c). The glioma vasculature in the WT was more disorganized than in the Vegfr2 Y949F/Y949F (Fig. 3d). Hotspots of fluorescent lectin leaking from perfused vessels (Fig. 3d, boxed area) correlated with high tumour uptake of Cadaverine-555 (Fig. 3e) in the WT compared with the mutant.

RIP1-TAg2:Vegfr2 $2^{Y 949 F / Y 949 F}$ leakage and metastatic spread. The consequence of the stabilized endothelial junctions in
Vegfr $2^{Y 949 F / Y 949 F}$ mice during tumour progression was further explored in the RIP1-TAg2 model. $\beta$-cells in the islets of Langerhans in RIP1-TAg2 mice express SV40 T antigen controlled by the rat insulin promoter, giving rise to multiple spontaneous pancreatic neuroendocrine tumours ${ }^{20}$ that seed metastases in the liver ${ }^{21}$. While there was no difference in the total tumour volume between the RIP1-TAg2:WT and RIP1-TAg2:Vegfr $2^{Y 949 F / Y 949 F}$ genotypes (Fig. 4a), the number of angiogenic islets, which are small lesions representing an early stage in tumour development, was significantly increased in RIP1-TAg2:Vegfr $2^{Y 949 F / Y 949 F}$ mice (Fig. 4b). Conversely, the RIP1-TAg2:Vegfr2 $2^{\text {Y949F/Y949F }}$ presented with fewer large $(>5 \mathrm{~mm})$ neoplastic islet tumours (Fig. $4 \mathrm{c}$ ). Tumour cells were found in close proximity to the vasculature in the tumour periphery suggesting metastatic spread of the a

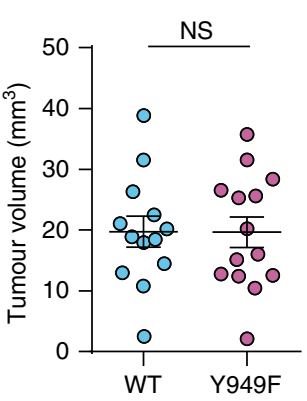

b

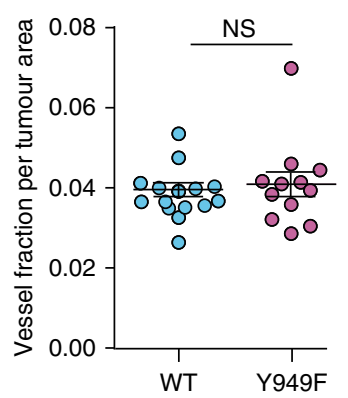

C

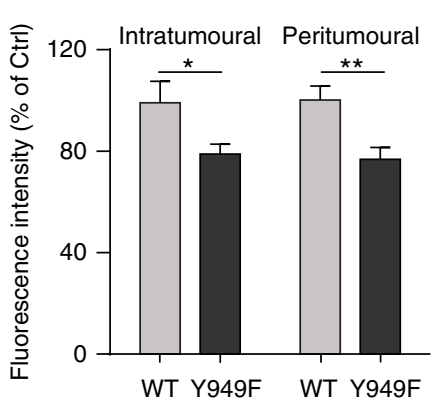


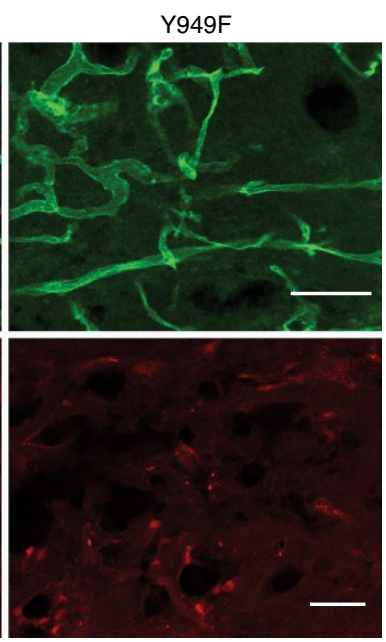

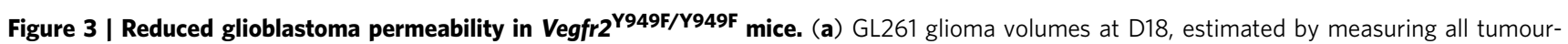


vessel density (area fraction per tumour area) at D18. $n=12-14$ mice per genotype. (c) Cadaverine-555 fluorescence intensity units estimated within the tumour (intratumoural) and at a 0-500- $\mu \mathrm{m}$ rim around the tumour border (peritumoural), Y949F normalized to WT. $n=8-11$ mice per genotype. (d) GL261 lectin-perfused vessels (lectin in green). Boxed area, typical point of leakage/disrupted barrier in the WT. (e) Cadaverine-555 (red) extravasation in WT and Y949F GL261 gliomas. Scale bar, $100 \mu \mathrm{m}$ (d) and $50 \mu \mathrm{m}$ (e). Data are presented as mean \pm s.e.m. Student's $t$-test, ${ }^{\star} P<0.05$, ${ }^{\star \star} P<0.01$. Experiments were performed three independent times. NS, not significant.

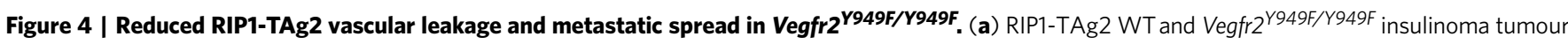
volumes at week 14. $n=19-21$ mice per genotype. (b) Angiogenic islet numbers at week 14. $n=14-16$ mice per genotype. (c) Tumour size distribution (1-5 and $>5 \mathrm{~mm}$ diameter), week 14. $n=17-19$ mice per genotype. (d) Tumour cells (SV40 T antigen ${ }^{+}$; green) and vessels (CD31+; red) in RIP1-TAg2 insulinomas (left), Arrows; individual tumour cells associated with vessels. Scale bar, $100 \mu \mathrm{m}$. SV40 T antigen ${ }^{+}$(green; upper) and haematoxylin-eosin (HE; lower) metastatic liver lesion (right). Scale bars, $50 \mu \mathrm{m}$. Number of liver metastases; mean per section, 30 sections per liver. $n=11-17$ mice per genotype. (e) Leakage of 30-nm microspheres (green; arrows) from VE-cadherin ${ }^{+}$tumour vessels (red). Scale bar, $50 \mu m$. (f) Microspheres area per VE-cadherin area from e. $n=10-11$ tumours per genotype. (g,h) VE-cadherin ${ }^{+}$(red) and podocalyxin ${ }^{+}$(green) RIP1-TAg2 tumour vasculature. Lower panels, magnification of boxed areas in upper panels. Arrows indicate typical disorganized podocalyxin in WT tumour vasculature. Scale bars, $50 \mu \mathrm{m}(\mathbf{g}), 5 \mu \mathrm{m}$ (h). (i) VE-cadherin area, \% of total area from $\mathbf{g} . n=15-17$ tumours per genotype. (j) Podocalyxin area, $\%$ of total area from $\mathbf{g} . n=15-17$ tumours per genotype. Data shown as mean \pm s.e.m. Student's $t$-test, ${ }^{\star} P<0.05,{ }^{\star \star} P<0.01, N S$, not significant. Data are pooled from three independent studies. 
insulinoma (Fig. 4d). Examination of livers showed that the number of SV40 T-positive liver metastases in the RIP1-TAg2:WT was significantly higher than in RIP1-TAg2:Vegfr2 ${ }^{Y 949 F / Y 949 F}$ mice at week 14 (Fig. 4d). We hypothesized that reduced metastatic spread in RIP1-TAg2:Vegfr2 ${ }^{\text {Y949F/Y949F mice could }}$ correlate with reduced vascular leakage. Tail vein-injected
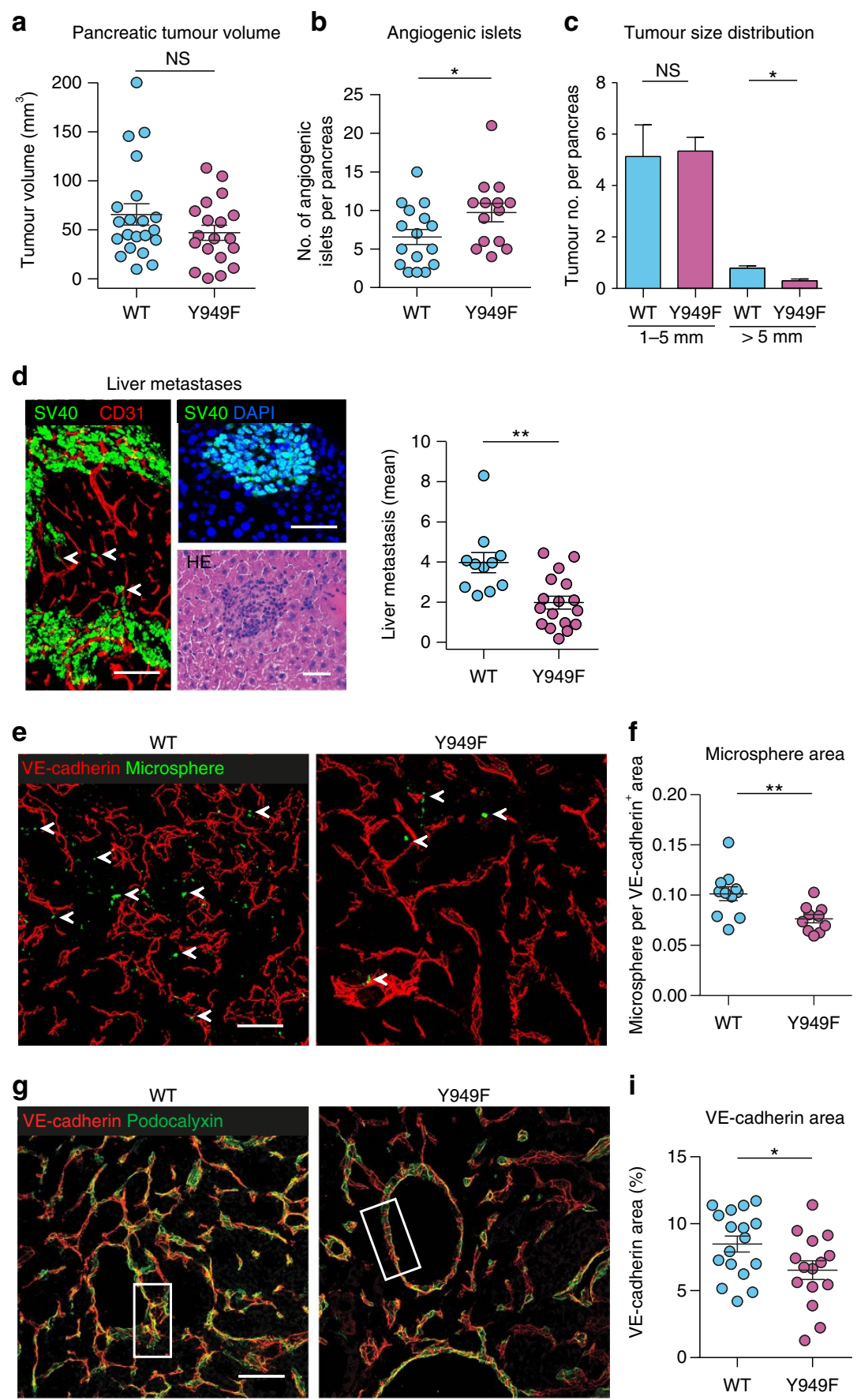

h

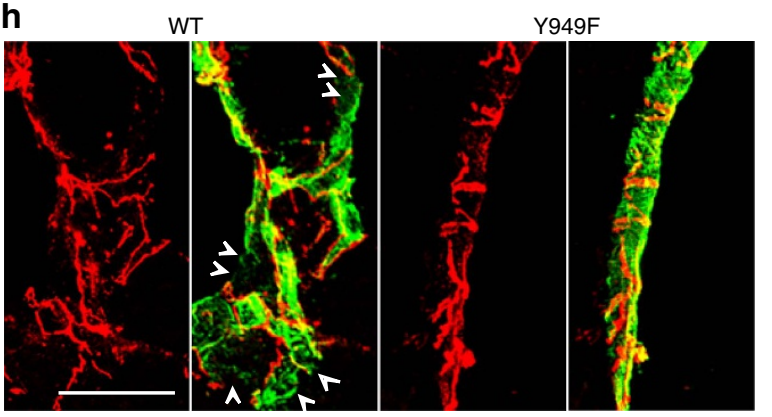

\section{j}

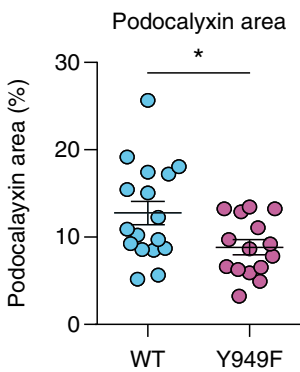


a

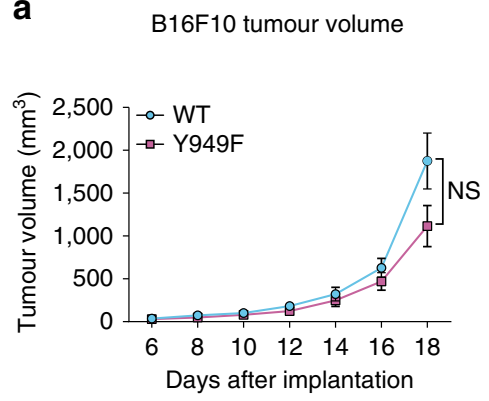

b

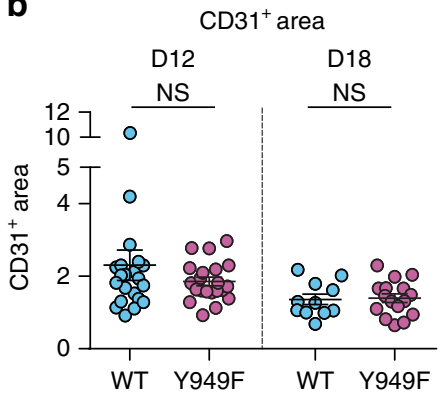

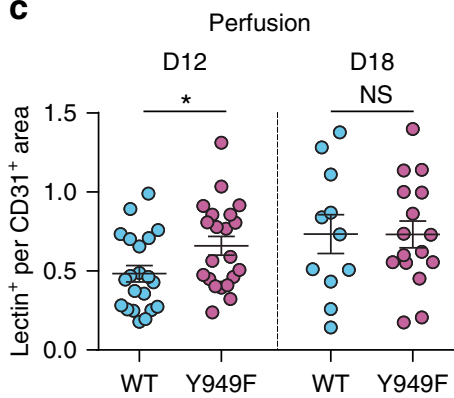

d
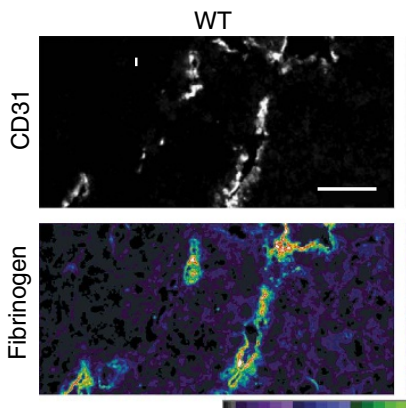
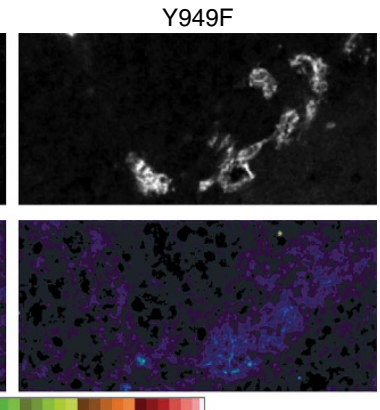

e

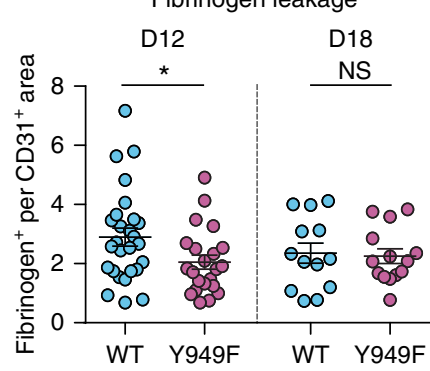

f

Tumour water content

g

Chemotherapy


$\mathbf{h}$ B16F10 ear tumour volume

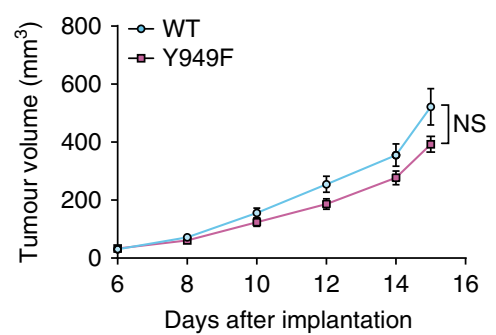

i



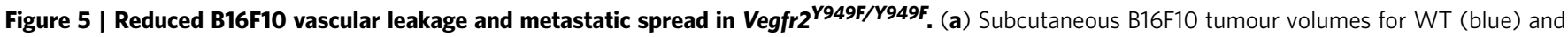
Vegfr2 Y949F/Y949F (Y949F; magenta) mice at different days after inoculation. $n=14-15$ mice per genotype. Repeated measures ANOVA: $P$ (time) <0.0001; $P($ genotype $)=\mathrm{NS}$. (b) $\mathrm{CD} 31^{+}$area per field in B16F10 tumours from WT and Y949F mice. $n=21$ mice per genotype at D12 and 11-16 mice per genotype at D18. (c) Area of tomato lectin-perfused vessels normalized to $\mathrm{CD} 31^{+}$area. $n=21$ mice per genotype, D12; and 11-16 mice per genotype at D18. ${ }^{\star} P<0.05$. (d) Tumour vasculature (upper panels; $\mathrm{CD} 31^{+}$vessels; white) and perivascular fibrinogen deposition (lower panels, heatmap from red, high, to blue, low) in

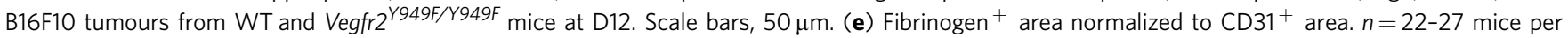
genotype at D12 and 13-14 mice per genotype at D18. Student's t-test, $P=0.0310$. (f) Oedema in B16F10 tumours estimated by weighing tumours before and after drying. Data show (wet weight - dry weight)/wet weight in \% at D12 and D18. $n=16-18$ mice per genotype at D12 and 19-24 mice per genotype at D18. Student's $t$-test, $P=0.0385$. (g) Tumour volumes at D12 of TMZ or vehicle (dimethylsulfoxide)-treated mice with B16F10 tumours, receiving treatment between D4 and D8 after inoculation. $n=12-15$ mice per group. Kruskal-Wallis test, $P=0.0289$. (h) B16F10 tumour volumes in mouse ear. $n=12-13$ mice per genotype. Repeated measures ANOVA: $P($ time $)<0.0001 ; P$ (genotype) $=$ NS. (i) Spontaneous B16F10 lung metastasis spreading to the lungs from primary B16F10 tumours in the ear. dsred-B16F10 metastases in four longitudinal sections of each left main lung lobe were counted. $n=12$ mice per genotype. Mann-Whitney $U, P=0.0028$. Data shown as mean \pm s.e.m. Dashed lines in $\mathbf{b}, \mathbf{c}, \mathbf{e}$ and $\mathbf{f}$ indicate that data sets from D12 and D18 were not compared statistically. ${ }^{\star} P<0.05,{ }^{\star \star} P<0.01$, NS, not significant. Experiments were performed three independent times (a-e,h-j) or two times $(\mathbf{f}, \mathbf{g})$,

and data were pooled. 
microspheres leaked from tumour vessels to a higher extent in RIP1-TAg2:WT than in RIP1-TAg2:Vegfr2 $2^{Y 949 F / Y 949 F}$ tumours (Fig. 4e,f). In the RIP1-TAg2:Vegfr2 $2^{Y 949 F / Y 949 F}$ tumour vasculature, VE-cadherin was preferentially localized at endothelial junctions and the VE-cadherin area was lower than in the WT (Fig. 4g-i). Moreover, staining for the sialomucin podocalyxin decorated the luminal endothelial surface in the RIP1-TAg2:Vegfr2 ${ }^{Y 949 F / Y 949 F}$ tumour vasculature while RIP1TAg2:WT tumours showed a chaotic podocalyxin staining pattern suggestive of altered apical-basal (luminal-abluminal) endothelial polarity (Fig. 4g,h,j) and therefore, disturbed vessel organization. The extent of vessel perfusion, pericyte coating and inflammatory cell infiltration (that is, CD45, F4/80 and macrophage mannose receptor (MMR)-positive cells did, however, not differ between RIP1-TAg2:WT and RIP1TAg2:Vegfr2 $2^{Y 949 F / Y 949 F}$ tumours at week 14 (Supplementary Fig. 3a-e).

B16F10 vessel leakage and metastatic spread. To further test the effect of the VEGFR2-Y949F mutation on vascular integrity in cancer, we chose to study subcutaneous B16F10 melanoma ${ }^{22}$. B16F10 tumour growth in the back skin was monitored continuously showing similar growth curves for the two genotypes (Fig. 5a). To monitor dynamic changes in tumour and vascular parameters, tumours were collected at two different time points after inoculation, at D12 (average tumour volume $200 \mathrm{~mm}^{3}$ ) and at D18 (average tumour volume $1.5 \mathrm{~cm}^{3}$ ) from WT and

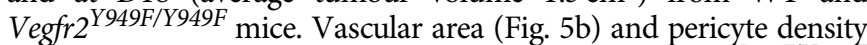
(Supplementary Fig. 3f) were similar in WT and Vegfr $2^{\text {Y949F/Y949F }}$ B16F10 tumours at D12 and D18. Vessel functionality as assessed by lectin perfusion was more efficient in the Vegfr $2^{\text {Y949F/Y949F }}$ tumour vasculature at D12 (Fig. 5c). At D18, vessel perfusion had improved in the WT and was similar to that of $V$ egf $r 2^{Y 949 F / Y 949 F}$ tumours. In spite of the improved perfusion, necrosis in the tumour was similar in the two genotypes (Supplementary Fig. 3g). Importantly, there were no differences in infiltration of $\mathrm{F} 4 / 80^{+}$, $\mathrm{CD}^{+} 5^{+}$and MMR-positive inflammatory leukocytes in the tumours at D12 (Supplementary Fig. 3h-j).

Vessel leakage in B16F10 tumours, determined by estimating the deposition of perivascular fibrinogen (Fig. $5 \mathrm{~d}$; quantified in e), was significantly lower in Vegfr2 ${ }^{Y 949 F / Y 949 F}$ tumours compared with WT tumours at D12. At D18, fibrinogen deposition was reduced in the WT tumours and similar between the two genotypes. Vascular leakage in tumours leads to oedema, which was estimated by weighing tumours before and after drying. The water content of Vegfr2 $9949 \mathrm{~F} / \mathrm{Y949F}$ tumours was significantly lower than in WT tumours at D12 (Fig. 5f) but not at D18. Altogether, these data indicate that the block in VEGFR2 pY949 signalling constrained vascular leakage and oedema in the tumour up to a critical point in tumour growth, occurring around D12. Thereafter, leakage and oedema were reduced irrespective of genotype, possibly due to an overall dysfunctional tumour circulation.

To test the effect of chemotherapy on tumours exhibiting different extents of vessel leakage and oedema, B16F10 tumourbearing mice were treated with a subtherapeutic dose of temozolomide (TMZ) or vehicle in one cycle for 5 consecutive days. Tumour growth in WT mice was resistant to the TMZ treatment at the given dose. In contrast, TMZ-treated

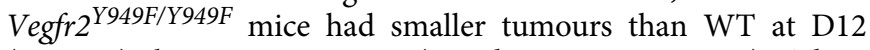
(Fig. 5g) but not at D18 (Supplementary Fig. 4a). Thus, the VEGFA-resistant vessel barrier phenotype in the Vegfr2 ${ }^{Y 949 F / Y 949 F}$ tumours correlated with an enhanced drug effect at D12, probably due to the lower oedema, and hence a lower interstitial tumour pressure, at this stage.
To study B16F10 metastasis, dsRed-B16F10 cells were inoculated intradermally in the ear. Tumour growth rate is constrained in the ear dermis, which we found correlated with an increased propensity to metastasize compared with when tumours grow unconstrained in the back skin. The primary tumour growth rate was similar between Vegfr2 ${ }^{Y 949 F / Y 949 F}$ and WT mice (Fig. 5h). Metastatic spread from the primary dsRed-B16F10 tumour to the lungs was however significantly reduced in the Vegfr $2^{Y 949 F / Y 949 F}$ mice (Fig. 5i). There was no correlation between the end point weight of the primary tumour and the number of metastatic foci (Supplementary Fig. 4b). We further tested the capacity of tail vein-injected dsRed-B16F10 tumour cells to colonize lungs and found no difference in lodging frequency between the genotypes (Supplementary Fig. 4c).

In vivo signalling in the Vegfr2 ${ }^{Y 949 F / Y 949 F}$ endothelium. To decipher signalling regulating VEGFA-induced vascular leakage in vivo, mice were subjected to tail vein injection of VEGFA or vehicle, and lungs were collected after different time periods of circulation (1-60 min). Lungs were chosen since they consist at least $50 \%$ of endothelial cells. Moreover, we used the two-tracer technique $^{23}$ to show that WT lung endothelial cells respond to VEGFA with increased transvascular leakage of iodinated albumin in vivo. The leakage rate in WT mice increased 2.5-fold after VEGFA injection in the tail vein, compared with vehicle (flow rate in response to VEGFA, $0.859 \pm 0.114$ versus vehicle, $0.334 \pm 0.126 \mu \mathrm{lmin}^{-1} \mathrm{~g}^{-1}$; s.e.m., $P=0.0278, n=3-4$ mice per condition).

VEGFA induced a very rapid and strong phosphorylation of VEGFR2 at Y1173 in WT and to a lower relative level in the Vegfr $2^{Y 949 F / Y 949 F}$ lung endothelium (Fig. 6a). Phosphorylation at Y949 was also high at $1 \mathrm{~min}$ after VEGFA injection in the WT, whereas it was absent in the Vegfr2 $2^{Y 949 F / Y 949 F}$ vasculature (Supplementary Fig. 5a). The lower pY1173 accumulation in the Vegfr $2^{Y 949 F / Y 949 F}$ could be due to the more extensive complex formation with the vascular endothelial protein tyrosine phosphatase (VEPTP). As shown in Supplementary Fig. 5b, there was a significantly higher extent of VEPTP/VEGFR2 complex formation in the Vegfr $2^{Y 949 F / Y 949 F}$ immunoprecipitates compared with WT VEGFR2 (two-way analysis of variance (ANOVA), genotype effect: $P=0.0037)$. VEPTP has been shown to restrict VEGFR2 tyrosine phosphorylation ${ }^{24}$.

Several signalling pathways regulate vascular permeability and leakage, including VEGFA-induced c-Src signalling 8,25 and histamine-induced phosphoinositide 3 kinase/Akt signalling ${ }^{26}$, which both induce phosphorylation of VE-cadherin and VE-cadherin internalization. We examined induction of pY658 VE-cadherin by immunoblotting of VEGFR2 immunoprecipitates from mouse lungs. We choose to study VE-cadherin in complex with VEGFR2 to monitor direct communication between the two. In the WT, the levels of pY658 VE-cadherin were already high in the PBS-injected mice in agreement with previous reports ${ }^{27}$, and remained high initially after injection of VEGFA, followed by a gradual decrease possibly due to the action of phosphatases, or to internalization and degradation of VE-cadherin (Fig. 6a). In the Vegfr $2^{Y 949 F / Y 949 F}$-derived samples, the pVE-cadherin level was low in the PBS condition and there was no induction with VEGFA injection (Fig. 6a).

Tyrosine phosphorylation of c-Src was difficult to detect in the VEGFR2 immunoprecipitates from lung lysate and was therefore examined in total lung lysates (Fig. 6b). In the WT samples, we detected an increased accumulation of pY418 c-Src only at the late time point, in agreement with previous data where c-Src activation was detected in lung lysates $20 \mathrm{~min}$ after tail vein injection of VEGFA ${ }^{8}$. In the Vegfr2 $2^{Y 949 F / Y 949 F}$ mouse lungs, 
a
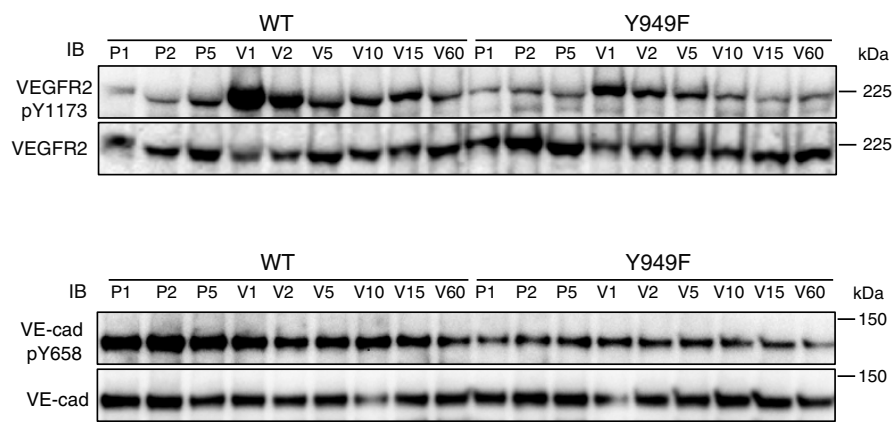

b
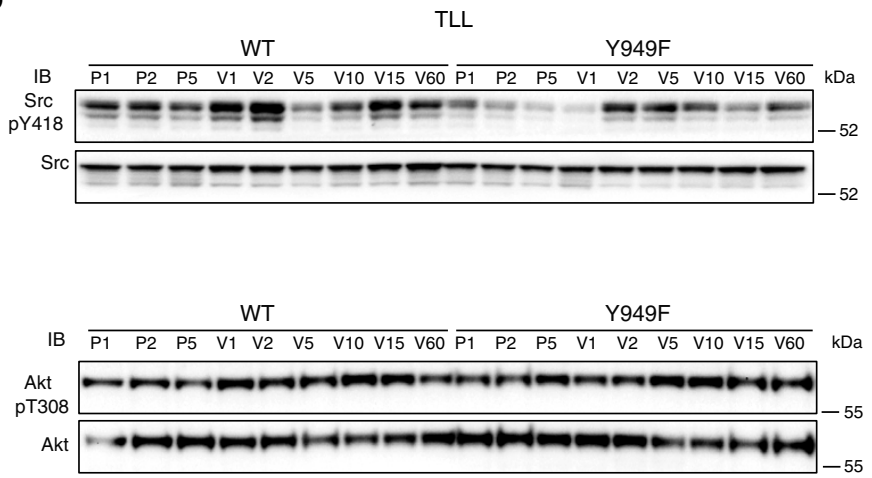

C

IP: VEGFR2, VEGFR2 activity
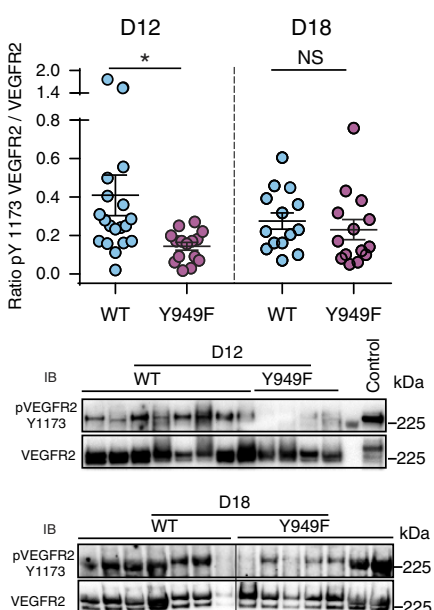

d
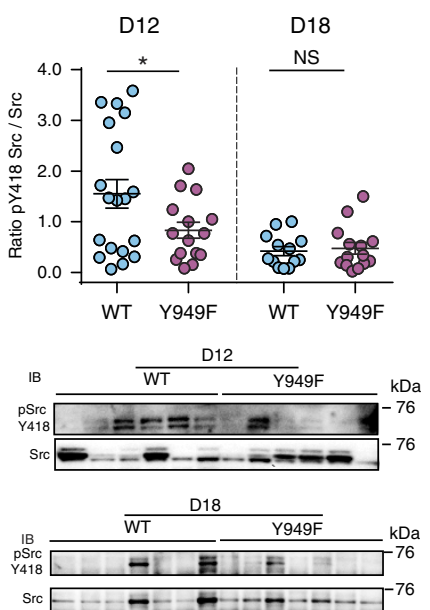
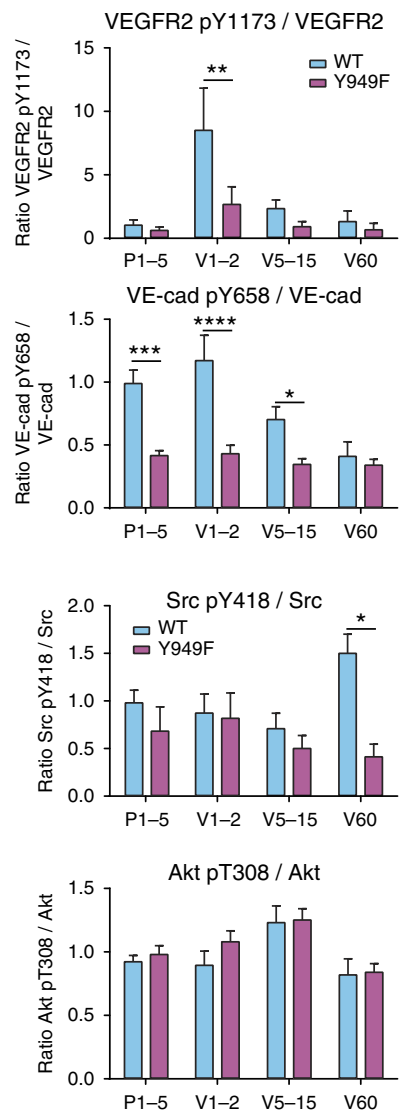

e

IP: VEGFR2, VEPTP complex
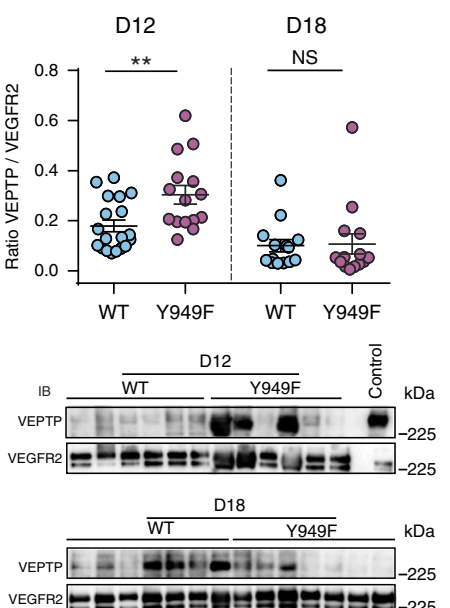

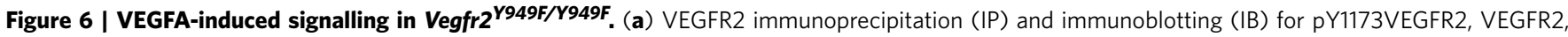
pY658VE-cadherin and VE-cadherin from lung lysates after tail vein injection of PBS (P) or VEGFA (V) in WT and Vegfr2 Y949F/Y949F (Y949F) mice, followed by circulation for different time periods (P1, P2, P5: PBS injection and circulation for 1, 2 or 5 min; V1, V2, V5, V10, V15, V60: VEGFA injection and circulation for $1,2,5,10,15$ or $60 \mathrm{~min})$. Quantification shown to the right; $n=4$. Groups of early (V1-V2), intermediate (V5, V10, V15) and late (V60) time points were quantified. Phosphoform/total protein band densities were normalized to the mean of all PBS samples. Two-way ANOVA, upper (p1173VEGFR2):

$P($ genotype $)=0.032, P($ time $)=0.0009$; lower $(V E$-cadherin): $P$ (genotype) $<0.0001, P($ time $)=0.0049$. (b) VEGFA-induced accumulation of pY418 Src (upper) and pT308 Akt (lower) in total lung lysates (TLL) from Vegfr2 Y949F/Y949F (Y949F) mice. Quantification as in a, relative to the unphosphorylated c-Src and Akt pools and normalized to mean PBS values. Two-way ANOVA, upper (Src): P(genotype) =0.0137, $P$ (time) $=$ NS; lower (Akt):

$P($ genotype $)=$ NS, $P$ (time) $=0.0013$. (c) Quantification (top) of IB (bottom) for pY1173VEGFR2 and VEGFR2 in VEGFR2 immunoprecipitates (IP) from B16F10 tumours at D12 and D18 after inoculation of WT and Vegfr2Y949F/Y949F (Y949F) mice. $n=19 /$ WT D12, 14/WT D18, 15/Y949F D12 and 14/Y949F D18. (d) Quantification (top) of IB (bottom) for pY418Src and c-Src in VEGFR2 immunoprecipitates (IP) from tumour lysates as in c. $n=19 /$ WT D12, 13/ WT D18, 14/Y949F D12 and 14/Y949F D18. (e) Quantification (top) of IB (bottom) for VEPTP and VEGFR2 on VEGFR2 immunoprecipitates (IP) from tumour lysates as in c. $n=19 /$ WT D12, 14/WT D18, 15/Y949F D12 and 14/Y949F D18 tumours. Data shown as mean \pm s.e.m. Dashed lines in c-e indicate that data sets from D12 and D18 were not compared statistically. In a,b two-way ANOVA, with Sidak's post hoc test; in c-e Student's $t$-test. ${ }^{\star} P<0.05$, ${ }^{\star \star} P<0.01,{ }^{\star \star \star} P<0.0001,{ }^{\star \star \star \star} P<0.00001$. Experiments were performed four independent times. 



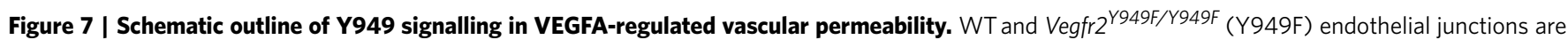
shown (upper). In the WT, the junction is active, that is, VE-cadherin is not engaged in homophilic interaction, and VEGFA signalling results in c-Src activation at junctions. In the Y949F mutant, VEGFR2 remains in complex with VEPTP and VE-cadherin, promoting junctional quiescence. c-Src may be activated but not at junctions. Lower schematics shows that junctional activation in the WT results in extravasation of cells and molecules, causing tissue oedema as well as metastatic spread via tumour cell intravasation into the blood stream (cells indicated in green). In the Y $949 \mathrm{~F}$ mutant, extravasation and oedema are blocked and metastatic spread is suppressed, while inflammatory cell extravasation is unaffected.

tyrosine phosphorylation of c-Src Y418 increased with VEGFA during the early phase but did not accumulate in the later phase of signalling (Fig. 6b, upper). Thus, the profiles of VEGFAinduced, endothelial c-Src phosphorylation differed between the WT and Vegfr $2^{Y 949 F / Y 949 F}$ mice. On the other hand, pT308AKT, examined in total lung lysates, accumulated with relatively slow and similar kinetics in the WT and Vegfr2 $2^{\text {Y949F/Y949F }}$ lungs in response to VEGFA injection (Fig. 6b, lower). These data indicate that the VEGFR2-Y949F mutation interfered with c-Src activation and pathways downstream of $\mathrm{c}$-Src, including VE-cadherin phosphorylation at Y658.

VEGFA signalling in B16F10 tumours was examined to understand how leakage was regulated in a tumour context. Immunoblotting of lysates from individual tumours showed that the pY1173/VEGFR2 ratio was significantly higher at D12 in tumours from WT mice compared with Vegfr2 $2^{Y 949 F / Y 949 F}$ mice (Fig. 6c; lower part shows representative immunoblot), correlating with the VEGFA signalling data from lung lysates (Fig. 6a). At D18, the pVEGFR2/VEGFR2 ratios were similar between the genotypes. The results on the pVEGFR2/VEGFR2 levels are likely to reflect the status in the tumour vasculature, as B16F10 cells do not express VEGFR2 (ref. 28). Immunoblotting for pY418Src in the VEGFR2 immunoprecipitates from tumour lysates showed a significantly higher $\mathrm{pSrc} / \mathrm{Src}$ ratio in the D12 tumours from WT mice compared with Vegfr $2^{Y 949 F / Y 949 F}$ mice (Fig. 6d; lower part shows representative blot). At D18, there was no difference between the genotypes in the extent of pSrc accumulation.
The restriction in VEGFR2 signalling in tumours from the Vegfr $2^{Y 949 F / Y 949 F}$ mice at D12 may involve dephosphorylation of VEGFR2 by VEPTP, as we found increased levels of VEGFR2/ VEPTP complexes in the Y949F condition (Fig. 6e; lower part shows representative blot). VEPTP expression levels in D12 tumours did not differ between the genotypes (Supplementary Fig. 5c). At D18, VEGFR2/VEPTP complex formation was similar between WT and Vegfr $2^{Y 949 F / Y 949 F}$-derived tumours (Fig. 6e). For uncropped versions of all blots presented in this study, see Supplementary Figs 6-13.

We conclude that VEGFA-induced leakage (see Fig. 7 for a schematic outline of VEGFA signalling regulating vascular permeability/leakage) can be restricted during a window of time of B16F10 tumour growth, by suppressing the pY949 pathway. Moreover, the data indicate that VEGFA signalling is dynamically regulated by VEPTP in solid tumours.

\section{Discussion}

Here we show that a specific arrest in VEGFA-induced vascular permeability leading to attenuation of molecular extravasation in organs such as the trachea and skin is compatible with normal vascular development, morphology and function. The Vegfr $2^{\text {Y949F/Y949F }}$ mouse displayed reduced vascular leakage in several orthotopic tumour models and a significant reduction in metastatic spread of insulinoma and melanoma (see Fig. 7 for a schematic summary of the results). The enhanced vascular barrier in the Vegfr2 $2^{Y 949 F / Y 949 F}$ mouse led to reduced tumour oedema, 
allowing for increased chemotherapeutic efficacy. While our data strongly indicate that signalling via pY949-VEGFR2 regulates c-Src activation and VE-cadherin phosphorylation and therefore adherens junction integrity, we do not rule out the involvement of transendothelial pores/vesiculovacuolar organelles in VEGFA-induced vascular permeability ${ }^{29}$.

Induced vascular permeability, also denoted vascular hyperpermeability or vascular leakage, may be acute or chronic ${ }^{30}$. The best studied factors regulating vascular leakage include VEGFA and inflammatory cytokines such as histamine and bradykinin ${ }^{31}$. An implication from the current study is that VEGFA dominates over inflammatory cytokines in regulating molecular extravasation, in the models studied here. VEGFA was originally identified as a vascular permeability factor ${ }^{30}$, but the physiological role of vascular permeability regulated by VEGFA/ VPF has remained unclear. Dvorak and colleagues implicated vascular permeability in the formation of a provisional matrix for the growth of new vessels ${ }^{32}$. Our data do not support an essential role for the VEGFA-driven pathway regulated by VEGFR2 pY949 signalling, in angiogenesis.

Cheresh and coworkers have shown the critical role for c-Src-pathways in regulation of VEGFA-induced vascular permeability $^{25}$. Our work extends these data by implicating pY949-VEGFR2/TSAd in regulation of c-Src pY418, VE-cadherin pY658 and the stability of adherens junctions. VE-cadherin is constitutively tyrosine phosphorylated in postcapillary venules compared with arteries/arterioles and regulated through flowinduced activation of c-Src, as judged from immunofluorescent staining of different capillary beds ${ }^{27}$. In this study, we noted a marked reduction in VE-cadherin Y658 phosphorylation in the Vegfr $2^{\mathrm{Y} 949 F / \mathrm{Y} 949 \mathrm{~F}}$ lung lysates compared with WT, in agreement with the altered c-Src phosphorylation profile (Fig. 6a,b) and with the persistence of adherens junctions in response to VEGFA, in vivo and in vitro (Fig. $1 \mathrm{~g}-\mathrm{j}$ ). However, the complex biology of c-Src precludes a direct cause-effect relationship between c-Src pY418 phosphorylation and VE-cadherin/adherens junction stability. We have previously shown that pY418 Src accumulates with relatively slow kinetics in vivo and that it translocates to junctions in complex with the adaptor molecule $\mathrm{TSAd}^{8}$. It is possible that the dynamics of c-Src activation differs between different subcellular compartments such as junctions and focal adhesions. Thus, at focal adhesions, c-Src activity is induced as a consequence of cell adhesion-dependent focal adhesion kinase activation, which also affects vascular permeability most likely by orchestrating cell shape ${ }^{33}$. In agreement with the data presented here, focal adhesion kinase (Ptk2) gene knockout decreases tumour spread ${ }^{34}$. Owing to technical constraints imposed by the quality of commercial c-Src antibodies, we have not been able to distinguish different subcellular pY418 Src pools in vivo. However, accumulation of pY418 Src in relation to the total c-Src pool was decreased both in Vegfr ${ }^{\text {Y } 949 F / Y 949 F}$ lung endothelial cells and in B16F10 tumours growing in mutant mice.

VEGFA-induced accumulation of pT308 Akt was similar in the WT and mutant Y949F VEGFR2 conditions. Akt plays an important role in vascular permeability induced by inflammatory cytokines $^{26}$. Akt is critical for the activation of endothelial NO synthase (eNOS) in vivo, by phosphorylation on Ser1176 (ref. 35), resulting in increased NO production. NO, a potent regulator of the vascular tone, mediates vasodilation by stimulating soluble guanylyl cyclase and increasing cyclic GMP in smooth muscle cells $^{36}$. In this study, we recorded transiently reduced blood pressure indicative of vasodilation in response to VEGFA in the descending aorta (Fig. 2j) in both WT and Vegfr2 ${ }^{\mathrm{Y} 949 F / Y 949 F}$ mice. This result together with the full activation of Akt, judged from the pattern of pT308 Akt accumulation in the mutant, indicate that the NO pathway is intact in the Vegfr2 $2^{Y 949 F / Y 949 F}$ mouse. eNOS has previously been implicated in tumour vascular permeability, and inhibition of eNOS activity by a small caveolin-derived peptide (cavtratin) leads to suppressed tumour vascular permeability and reduced primary tumour growth without affecting blood flow and angiogenesis ${ }^{37}$. In contrast, while metastatic spread was suppressed in the Vegfr2 ${ }^{\text {Y949F/Y949F }}$ mouse, primary tumour growth was only modestly affected.

We noted reduced phosphorylation of VEGFR2 at phosphosite Y1173 in the Vegfr2 ${ }^{Y 949 F / Y 949 F}$ lungs and in D12 B16F10 melanoma. Mutation of the human tyrosine residue, Y951 (corresponding to mouse Y949), in a purified recombinant intracellular VEGFR2 domain interferes with protein folding ${ }^{38}$. Whether protein folding is affected also in the full-length VEGFR2 remains to be shown. We noted that the level of phosphorylation of the full-length mouse VEGFR2-Y949F mutant relative to WT VEGFR2 varied during tumour progression. Thus, in B16F10 tumours, the relative level of pY1173 in WT VEGFR2 was higher than for VEGFR2-Y949F at an early stage, whereas the levels were similar at later stages of tumour growth. We propose that the reduced VEGFR2 phosphorylation in the Vegfr2 ${ }^{Y 949 F / Y 949 F}$ lungs in D12 B16F10 tumours at least in part was a consequence of sustained VEGFR2/VEPTP complex formation. VEPTP is a receptor-type phosphotyrosine phosphatase specifically expressed in endothelial cells and known to have VE-cadherin and Tie2 as direct substrates $^{39}$. VEGFR2 is an indirect substrate for VEPTP, resulting in dephosphorylation of VEGFR2 in complex with Tie2 (ref. 24). Here we confirm and extend the data on the important role of VEPTP as an in vivo regulator of VEGFR2 activity. VEGFR2/VEPTP complex formation correlated with VEGFR2 pY1173 levels and the extent of oedema. Thus, B16F10 melanomas in WT mice showed low VEGFR2/VEPTP complex formation, high p1173 VEGFR2 levels and prominent oedema. In contrast, melanomas in the Vegfr $2^{Y 949 F / Y 949 F}$ mice displayed high VEGFR2/VEPTP complex formation, low p1173 pVEGFR2 levels and reduced oedema. With time, VEPTP/VEGFR2 complex formation decreased in tumours from both WT and Vegfr $2^{Y 949 F / Y 949 F}$ mice. Still, the VEGFR2 and c-Src activities were suppressed in the late stage of tumour growth in WT mice, showing a restriction in VEGFA signalling independent of VEPTP. The gradual progression towards lower VEGFR2 phosphotyrosine levels over time is in agreement with models for resistance towards anti-VEGFA neutralizing therapy in cancer ${ }^{40}$.

The extent of tumour oedema correlates to the blood supply, the volume of blood entering the tumour and the lymphatic drainage $^{41}$. Tumour blood supply decreases as the tumour mass increases ${ }^{42}$, most likely due to a progressive deterioration of the tumour vasculature with eventually fewer functional vessels. Tumours in Vegfr2 $2^{\text {Y } 949 F / Y 949 F}$ mice were less oedematous, which we attribute to the higher vessel integrity in the mutant. Excess and persistent extravasation increases the interstitial pressure and impairs delivery of therapeutics to the tumour tissue 4 . In agreement, the B16F10 tumour-bearing Vegfr2 ${ }^{Y 949 F / Y 949 F}$ mice were more responsive to treatment with chemotherapy. Complete sealing of junctions to VEGFA, as well as to inflammatory cytokines, as a result of expression of a VE-cadherin/ $\alpha$-catenin fusion protein, leads to a partial reduction in inflammatory cell extravasation ${ }^{43}$. In contrast, restricting VEGFA-induced permeability alone as in the Vegfr $2^{Y 949 F / Y 949 F}$ genotype, failed to suppress inflammatory cell infiltration in RIP1-TAg2 insulinomas (Supplementary Fig. 3c-e) and B16F10 melanomas (Supplementary Fig. $3 \mathrm{~h}-\mathrm{j}$ ). While we do not exclude that acute inflammation or other aspects of inflammation not studied here are affected by the VEGFR2 mutation, our data suggest that there is no direct relationship between the capacity to spread metastasis 
and primary tumour inflammation. Moreover, lodging of circulating tumour cells was unaffected by the mutation (Supplementary Fig. 4b) suggesting that the stable Vegfr2 ${ }^{Y 949 F /}$ Y949F junctions may restrict the ability of tumour cells to enter but not exit the circulation. The caveats of the experimental metastasis model ${ }^{44}$ (that is, injection of tumour cells in the tail vein followed by lodging of tumour cells in the lung) compared with spontaneous metastasis, limits conclusions however.

Possibly, factor(s) other than VEGFA may contribute to extravasation of $\mathrm{B} 16 \mathrm{~F} 10$ cells in the lung; indeed, the


permeability-inducing factors than VEGFA such as histamine. It is pertinent to ask whether the reduced metastatic spread in the Vegfr2 ${ }^{Y 949 F / Y 949 F}$ tumours can be attributed directly to the VEGFA-resistant Y949F junctions, that is, do tumour cells intravasate into the circulation via junctions? Alternatively, were other parameters, established as a consequence of the nonleaky junctions and the reduced oedema, more important? As we did not record significant changes compatible with vascular normalization ${ }^{4}$, that is, in vascular density, pericyte coating, necrosis or inflammation in the B16F10 melanomas from WT and mutant mice, we propose that the Vegfr2 $2^{Y 949 F / Y 949 F}$ phenotype primarily depends on the VEGFA-resistant junctions.

In conclusion, our data suggest that specifically suppressing VEGFA-induced vascular permeability without attenuation of vital aspects of VEGFA biology, for example, endothelial survival and neoangiogenesis, may offer an attractive strategy in future drug development to reduce oedema, to improve therapeutic efficacy and to suppress metastatic spread in cancer.

\section{Methods}

Vegfr2 ${ }^{\text {Y949F/Y949F }}$ mouse model. A mouse model with knock-in of phenylalanine (F) to replace the tyrosine (Y) at position 949 (Y951 in the human) of VEGFR2 (designated Vegfr2 $2^{Y 949 F / Y 949 F}$ ) in a mixed 129S6/C57BL/6 was created using VelociGene technology (Regeneron Pharmaceuticals, New York, USA) ${ }^{11}$. The Vegfr $2^{\text {Y } 949 F / Y 949 F}$ mouse was backcrossed onto the C57BL/6 background for more than 10 generations and maintained through crossing of $V e g f r 2^{Y 949 F / Y 949 F}$ heterozygotes. All data shown are based on comparison of $V e g f r 2^{Y 949 F / Y 949 F}$ and WT littermates obtained from heterozygote crossings in experiments repeated at least three times. The Uppsala University board of animal experimentation approved all animal work; efforts were done to minimize suffering as supervised by the University veterinarian. Mice were anaesthetized with ketamine/xylazine, avertin or isoflurane (Isoba, 3\% induction and 1.5\% maintenance).

Statistical analysis. For parametric comparison, Student's $t$-test was performed to compare means between two groups. One-way ANOVAs were performed to compare $>2$ treatment groups and two-way ANOVA was used when two factors were involved, for example, treatment and genotype. Repeated measures ANOVA was used for tumour growth curves. Multiple comparisons post hoc tests were chosen based on how many group comparisons were made. When all groups were compared with each other, Tukey's Honestly Significant Difference (HSD) test was used, while Sidak's test was used when individual time points were compared with each other. Non-parametric Mann-Whitney $U$-test/Wilcoxon sign-rank test (for paired comparison) was used to compare two groups, while Kruskal-Wallis test followed by Dunn's multiple comparisons was used for $>2$ group-wise comparisons. GraphPad Prism 6.0 was used for statistical analyses. The threshold for statistical significance was set at $\alpha=0.05\left({ }^{\star} P<0.05,{ }^{* *} P<0.01\right.$ and $\left.{ }^{* *} P<0.0001\right)$.

Antibodies and growth factors. All antibodies used in this study, their origin and working concentrations are listed in Supplementary Table 1.

Recombinant mouse VEGFA164 was purchased from Peprotech EC Ltd (Rocky Hill, NJ, USA) and used for in vitro analyses. Dog VEGFA164, which is $99 \%$ identical to mouse VEGFA, was used for in vivo analyses and was produced at the Paul-Scherrer Institute, Villigen, Switzerland. In vitro tests showed no difference in VEGFR2-stimulating activity of commercial mouse VEGF and in-house-produced dog VEGF. Histamine was from Sigma-Aldrich.

Orthotopic mouse glioma model. Mice (homozygous Vegfr2 ${ }^{Y 949 F / Y 949 F}$ and WT littermates) were inoculated with GL261 glioblastoma cells (a kind gift from Geza Safrany, NRIRR, Budapest). Cells were not verified but tested negative for mycoplasma using the Mycoplasma detection kit (Lonza). GL261 is a chemically induced murine cell line, which when inoculated orthotopically has many features reminiscent to human glioma ${ }^{18}$. Cells were cultured in DMEM Glutamax medium containing 10\% FCS, washed and resuspended in PBS for inoculation. Mice were anaesthetized with isoflurane (Isoba, $3 \%$ induction and $1.5 \%$ maintenance). A small incision was made rostrocaudally along the midline of the scalp. A small hole was drilled at stereotaxic coordinates $+1.1 \mathrm{~mm}$ anterior and $2.1 \mathrm{~mm}$ lateral to bregma. A volume of $0.5 \mu \mathrm{l}$ of cells $\left(1.5 \times 10^{5}\right.$ cells per $\left.\mu \mathrm{l}\right)$ were slowly injected using a Hamilton microsyringe with a $33 \mathrm{G}$ needle at $2.9 \mathrm{~mm}$ depth below the dura. The needle was left in place for $3 \mathrm{~min}$ before slow retraction over $2 \mathrm{~min}$. The wound was closed using 6-0 resorbable suture, and analgesia carprofen (Rimadyl Vet, Orion Pharma, Sweden; $50 \mu \mathrm{g}$ per $20 \mathrm{~g}$ mouse) was injected subcutaneously on the back Tumours were left to grow for 18 days. On the last day, mice were intravenously injected with $150 \mu \mathrm{g}$ per $20 \mathrm{~g}$ mouse of Alexa-555-Cadaverine (Life Technologies) together with $100 \mu \mathrm{g}$ of biotinylated tomato lectin in PBS. After $15 \mathrm{~min}$ of circulation, mice were killed using an overdose of ketamine/xylazine (see above) and transcardially perfused with physiological saline followed by cold $4 \%$ paraformaldehyde (PFA). Brains were postfixed for $4 \mathrm{~h}$ and transferred to $25 \%$ sucrose for cryoprotection. Brains were coronally cut on a vibratome at $80 \mu \mathrm{m}$ thickness and mounted on cover glass in series to cover the entire volume of the tumour. Tumour volume was estimated using haematoxylin-and-eosin-stained sections throughout the brain. Representative sections were immunostained for lectin (Streptavidine-Alexa-488) and nuclei (Hoechst). The two largest (mid) sections of each tumour were used for quantification of vessel density and Cadaverine-555 accumulation in the brain, indicating abnormal leakage.

RipTag spontaneous endocrine tumour and metastasis model. Vegfr2 ${ }^{Y 949 F / Y 949 F}$ mice were crossed with RIP-Tag2 mice on the $\mathrm{C} 57 \mathrm{Bl} / 6$ background. Vegfr $2^{Y 949 F / Y 949 F}$ heterozygous offspring was bred to obtain RIP-Tag2:WT and


mixed gender aged 14 weeks. Islets larger than $1 \times 1 \mathrm{~mm}$ were defined as Riptag tumours, and overtly red islets $<1 \times 1 \mathrm{~mm}$ were defined as angiogenic islets as assessed under a stereological microscope. Tumours and pancreas including angiogenic islets were sectioned and stained to analyse vessel characteristics. For quantification of metastases, right liver lobes were sectioned in their entirety. In all, 30 consecutive sections were examined for score of metastases based on a cluster of at least 25 cells, which were confirmed by SV40-Tag staining. Average numbers of liver metastases per section was calculated.

B16F10 subcutaneous primary tumour and metastasis model. B16F10 cells (American Type Culture Collection) were not validated after purchase but retained melanin production and were regularly tested negative for mycoplasma using the Mycoplasma Detection Kit (Lonza). Cells $\left(0.5 \times 10^{6}\right.$ per $\left.100 \mu \mathrm{l}\right)$ were injected subcutaneously in the mouse back skin. Tumour size was measured with a caliper every other day from D6 and tumours were collected at D12 or D18 after implantation. Tumour wet and dry weights were measured to determine water content/oedema in the tumours, calculated (tumour wet weight - tumour dry weight)/tumour wet weight and given as percentage (\%). Tumour vessel characteristics were analysed after injection of FITC-Lectin (Sigma) in the tail vein, followed by 20 -min circulation and then, a washout with PBS via the left cardiac ventricle. Snap-frozen tumours were sectioned, stained and imaged using a Zeiss Axioimager. Quantification of vessel parameters was done using ImageJ (National Institutes of Health) in a blinded fashion. To score for metastatic spread, lentivirus-transduced DsRed-expressing B16F10 cells (kindly provided by Professor David D. Schlaepfer Department of Pathology, La Jolla, CA) were injected in the mouse right ear dermis $\left(4 \times 10^{4}\right.$ cells in $10 \mu \mathrm{l}$ growth factor-depleted Matrigel; BD). Tumour volume was measured with a caliper every other day. At D15, mice were perfused with PBS via the left cardiac ventricle and lungs were removed, fixed and frozen. For quantification of metastases, the left large lung lobe from each mouse was sectioned in its entirety. Lung metastases were enumerated by counting DsRed-positive lesions from confocal microscopy in four sections spaced $100 \mu \mathrm{m}$ apart, in each specimen.

Microsphere extravasation in mouse trachea and Riptag tumours. Twenty microlitres of $30 \mathrm{~nm}$ Fluoro-Max Green Aqueous Fluorescent microspheres (Thermo Scientific, Fremont, CA, USA) together with $80 \mu \mathrm{l}$ solution of VEGFA164 (5 $\mu \mathrm{g}$ per $20 \mathrm{~g}$ body weight) or PBS were administered by tail vein injection. Mice were transcardially perfused using 1\% PFA in PBS $1 \mathrm{~h}$ later. For histamine-induced microsphere extravasation, $20 \mu \mathrm{l}$ of $30 \mathrm{~nm}$ fluorescent microspheres was injected together with histamine solution $(80 \mu \mathrm{l} ; 1.25 \mathrm{mg}$ per $20 \mathrm{~g}$ body weight). Perfusion with $1 \%$ PFA was performed after 2 min circulation ${ }^{45}$. Tracheas were dissected and fixed in $1 \%$ PFA in PBS for an additional $2 \mathrm{~h}$, stained with endothelial cell markers to visualize the vasculature. To observe microsphere extravasation in Riptag tumours, $100 \mu \mathrm{l}$ of 30 -nm fluorescent microspheres was administered by tail vein injection. Intravascular microspheres were removed by PBS perfusion via the left ventricle $1 \mathrm{~h}$ later. Riptag tumours were postfixed in $2 \%$ PFA for $6 \mathrm{~h}$ and kept in $30 \%$ sucrose overnight at $4{ }^{\circ} \mathrm{C}$ followed by embedding in cryosectioning medium. Tumours were sectioned and stained for endothelial cell markers. Imaging was performed using a Zeiss700 confocal microscope or a Zeiss 710 Structured Illumination microscope.

TMZ chemotherapy. B16F10 cells $\left(0.5 \times 10^{6}\right.$ per $\left.100 \mu \mathrm{l}\right)$ were injected subcutaneously in the mouse back skin. From D4 to D8, TMZ dissolved in 
dimethylsulfoxide to a final concentration of $2 \%$, was administered i.p. every day at $10 \mathrm{mg} \mathrm{kg}^{-1}$, a dose established to have no effect on B16F10 growth in WT mice from test studies. Control mice were treated with a similar volume of $2 \%$ dimethylsulfoxide. Tumour size was measured with a caliper every other day from D6 until D18 after implantation.

\section{DsRed-expressing B16F10 cell lung colonization. DsRed-expressing B16F10} cells (1 million in $100 \mu \mathrm{lPBS}$ ) were injected into mouse tail vein. At $48 \mathrm{~h}$, mice were perfused with PBS via the left cardiac ventricle, and lungs were removed, fixed and frozen. Left lung lobes were sectioned in their entirety. Tumour cell foci were enumerated by counting DsRed-positive lesions in confocal microscopy in two sections spaced $400 \mu \mathrm{m}$ apart and five fields per section.

Miles assay. Mice (females, 6-8 weeks) were subjected to i.p. injection with pyrilamine maleate salt ( $4 \mathrm{mg}$ per $\mathrm{kg}$ body weight in $0.9 \%$ saline, Sigma) to inhibit unspecific histamine release (for example, by pulling the skin), 30 min before Evans' blue tail vein injection ( $100 \mu \mathrm{l}$ \% Evans blue in sterile saline, Sigma).

This was followed by intradermal injection of VEGF ( $100 \mathrm{ng}$ in $50 \mu \mathrm{l}$ per site) or sterile saline $10 \mathrm{~min}$ after Evans' blue administration. Thirty minutes after the VEGF injection, the dorsal skin was excised and Evans' blue was extracted by immersion in formamide buffer. Absorbance at $620 \mathrm{~nm}$ was measured using a spectrophotometer (EMax microplate reader, Molecular Devices, CA, US). Extravasated Evans' blue was determined from a standard curve and normalized to tissue weight (g).

Isolation of lung endothelial cells. Mouse lungs were collected at P10 or 3-4 weeks, minced and digested in $10 \mathrm{ml}$ Dulbecco's PBS medium containing $2 \mathrm{mg} \mathrm{ml}^{-1}$ collagenase type I (Sigma), for $1 \mathrm{~h}$ at $37^{\circ} \mathrm{C}$ with shaking, followed by filtration through a $70-\mu \mathrm{m}$ disposable cell strainer (BD Falcon). Cells were centrifuged at $400 \mathrm{~g}$ for $8 \mathrm{~min}$ at $4{ }^{\circ} \mathrm{C}$, suspended in cold PBS with $0.1 \%$ bovine serum albumin (BSA) and incubated with anti-rat immunoglobulin G-coated magnetic beads (Dynabeads sheep anti-Rat IgG, Invitrogen) pre-coupled with rat anti-mouse platelet/endothelial cell adhesion molecule-1 (PECAM-1; MEC13.3, BD Pharmingen, 553370) for $10 \mathrm{~min}$, with gentle agitation. Beads were separated from the solution with a magnetic particle concentrator (Dynal MPC-S, Invitrogen). The beads were washed five times with PBS, and endothelial cells were suspended in MV2 growth medium with the following supplements: $5 \mathrm{ng} \mathrm{ml}^{-1}$ endothelial growth factor; $0.2 \mu \mathrm{g} \mathrm{ml}^{-1}$ hydrocortisone; $0.5 \mathrm{ng} \mathrm{ml}^{-1}$ VEGFA; $10 \mathrm{ng} \mathrm{ml}^{-1}$ basic fibroblast growth factor; $20 \mathrm{ng} \mathrm{ml}^{-1}$ insulin-like growth factor-1 (Promocell); and $5 \%$ penicillin/streptomycin (Sigma). The purity of cells was $>95 \%$ as determined by staining for VE-cadherin. The isolated lung endothelial cells were cultured on slides pre-coated with gelatin. Cells were treated with or without $100 \mathrm{ng} \mathrm{ml}^{-1}$ mouse VEGFA164 for 10 min before fixation in 4\% PFA in PBS for $10 \mathrm{~min}$, permeabilized in $0.1 \%$ Triton X-100 in PBS for $30 \mathrm{~min}$, followed by immunofluorescent staining. Images were acquired using a Zeiss LSM700 confocal microscope $(\times 63$, numerical aperture 1.4 objectives).

\section{Immunoprecipitation and immunoblotting. For in vitro signal transduction} analyses, endothelial cells were isolated from lungs of P10 pups as described above, and cultured. Cells were treated with or without $100 \mathrm{ng} \mathrm{ml}^{-1}$ mouse VEGFA164 (PeproTech) for $8 \mathrm{~min}$ and lysed for subsequent immunoprecipitation and immunoblotting.

For in vivo signal transduction analyses, lung lysates were used from mice at 1 , $2,5,10,15$ or 60 min after injection of dog VEGFA164 in the tail vein. An equal volume of PBS was injected into control mice. B16F10 tumour lysates were obtained from mice at D12 or 18 after B16F10 cell implantation. Tissues were lysed in commercial RIPA buffer (ProteinSimple) followed by immunoprecipitation for VEGFR2 and immunoblotting, or immunoblotting of total lysates. For immunoprecipitation, $1.5 \mathrm{mg} / \mathrm{ml}$ tissue lysate were diluted in RIPA buffer $(20 \mathrm{mM}$ HEPES, pH 7.5, $150 \mathrm{mM} \mathrm{NaCl}, 1 \% \mathrm{NP} 40$ ) supplemented with protease inhibitors (Roche, 04693116001), phosphatase inhibitors (Thermo scientific, 88667), $1 \mathrm{mM}$ $\mathrm{NaF}, 1 \mathrm{mM} \mathrm{Na} 3 \mathrm{VO}$. Lysates were precleared by incubating with $25 \mu \mathrm{l}$ of Protein G Sepharose 4 Fast Flow beads (Ge Healthcare, 17-0618-01) per sample for $2 \mathrm{~h}$ at $4{ }^{\circ} \mathrm{C}$. Precleared lysates were incubated with $1.5 \mu \mathrm{g}$ VEGFR 2 antibody for $2 \mathrm{~h}$ at $4{ }^{\circ} \mathrm{C}$ and $25 \mu \mathrm{l}$ of Protein $\mathrm{G}$ beads were added and incubation continued for $1 \mathrm{~h}$ (lung lysates) and overnight (B16 tumor lysate and isolated lung endothelial cells). Sepharose beads were washed 5 times with $1 \mathrm{ml}$ RIPA buffer per sample without protease and phosphatase inhibitors and collected by centrifugation at $3000 \mathrm{Xg}$ for $1 \mathrm{~min}$ at $4{ }^{\circ} \mathrm{C}$. $30 \mu \mathrm{l}$ of $2 \mathrm{X}$ LDS sample buffer were added to each sample and heat at $70^{\circ} \mathrm{C}$ for $10 \mathrm{~min}$. Immunocomplexes were resolved on NuPAGE Novex $4-12 \%$ BisTris gels (Invitrogen) and processed for immunoblotting 8 . Full scans of all immunoblots are presented in Supplementary Figs 6-13.

Immunofluorescent staining. Eyes were removed and prefixed in 4\% PFA for $20 \mathrm{~min}$ at room temperature. After dissection, retinas were blocked overnight at $4{ }^{\circ} \mathrm{C}$ in $1 \%$ FBS (Gibco), 3\% BSA (Sigma) and $0.5 \%$ Triton X-100 (Sigma). Retina samples were incubated over night with primary antibodies in blocking reagent, followed by washing and incubation with Isolectin-B4 in Pblec ( $1 \mathrm{mM} \mathrm{MgCl}_{2}$, $1 \mathrm{mM} \mathrm{CaCl}_{2}, 0.1 \mathrm{mM} \mathrm{MnCl}_{2}$ and $1 \%$ Triton X-100 in PBS) overnight. Thereafter, samples were incubated with the appropriate secondary antibody for $2 \mathrm{~h}$ at room temperature, and mounted in fluorescent mounting medium (DAKO). Images were acquired using a Zeiss 700 confocal microscope. For analysis of the activation state of endothelial junctions in the retinal vasculature, the unbiased software $(\text { Matlab })^{15}$ was used.

Blood parameter measurements. The blood flow measurements were performed with a microsphere technique ${ }^{46}$ as described below: WT and Vegfr2 ${ }^{\text {Y949F/Y949F }}$ mice 3-4 months old were anaesthetized with isoflurane and placed on an operating table, where the animal's body temperature was maintained constant at $38^{\circ} \mathrm{C}$. A polyethylene tracheal tube was inserted to ensure free airways and polyethylene catheters were inserted into the ascending aorta, via the right carotid artery, and into the right femoral artery. The former catheter was connected to a pressure transducer (Physiologic Pressure Transducer; AD Instruments, Oxford, UK) to allow continuous monitoring of the mean arterial blood pressure, which was allowed to stabilize for $20-25 \mathrm{~min}$. Approximately $10^{5}$ microspheres (EZ-Trac; Triton Technology, San Diego, CA, USA) with a diameter of $10 \mu \mathrm{m}$ were injected for $10 \mathrm{~s}$ via the catheter with its tip in the ascending aorta. Starting $5 \mathrm{~s}$ before the microsphere injection, and continuing for a total of $60 \mathrm{~s}$, an arterial blood reference sample was collected by free flow from the catheter in the femoral artery at a rate of $0.10 \mathrm{ml} \mathrm{min}{ }^{-1}$. The exact withdrawal rate in each experiment was confirmed by weighing the sample.

Arterial blood was then collected from the catheter in the femoral artery and analysed for blood $\mathrm{pH}, \mathrm{pCO}_{2}, \mathrm{pO}_{2}$, oxygen saturation, $\mathrm{S}-\mathrm{HCO}_{3}^{-}$, base excess, $\mathrm{S}-\mathrm{Na}^{+}, \mathrm{S}-\mathrm{K}^{+}$, haematocrit and haemoglobin concentration, with an iStat (Abbott Scandinavia). The cassette used for this analysis requires $90 \mu \mathrm{l}$ of whole blood. Arterial blood was also used for the determination of blood glucose concentrations (Medisense Test Reagent Strips; MediSense) and for serum insulin determinations with ELISA (Rat Insulin ELISA; Mercoedia AB).

The animals were killed, and the organs were carefully dissected free from fat, blotted and weighed. The microspheres in the organs and the arterial blood reference sample were visualized by a freeze-thawing technique and counted by an observer blinded for the origin of the samples ${ }^{47}$.

The blood flow values were calculated according to the formula $Q_{\text {org }}=Q_{\text {ref }} \times N_{\text {org }} / N_{\text {ref }}$, where $Q_{\text {org }}$ is organ blood flow $\left(\mathrm{ml} \mathrm{min}^{-1}\right), Q_{\text {ref }}$ is withdrawal rate of the reference sample $\left(\mathrm{ml} \mathrm{min}^{-1}\right), N_{\text {org }}$ is the number of microspheres present in the organ and $N_{\text {ref }}$ is the number of microspheres present in the reference sample ${ }^{46}$.

Blood pressure measurements after VEGF administration. Mice (females, $\sim 15$ weeks) were anaesthetized by an intraperitoneal (i.p.) injection of avertin ${ }^{48}$. A polyethylene tracheal tube was inserted to ensure free airways, and a polyethylene catheter was inserted into the descending aorta, via the right femoral artery, and connected to a pressure transducer (AD Instruments) connected to a PowerLab system (AD Instruments. When the blood pressure had remained stable for $10 \mathrm{~min}$ an injection of $0.1 \mathrm{ml}$ of saline or VEGFA ( $5 \mu \mathrm{g}$ per $20 \mathrm{~g}$ body weight) was given in the right jugular vein. Mean arterial blood pressure was then registered for $15 \mathrm{~min}$.

E11.5 Hindbrain vascularization. Embryos at E11.5 were collected and fixed in $4 \%$ PFA overnight. Hindbrains were dissected the following day and thereafter, brains were permeabilized in a buffer containing $1 \%$ Triton $\mathrm{X}, 0.01 \mathrm{mM} \mathrm{CaCl} 2,0.1 \mathrm{mM}$ $\mathrm{MgCl}_{2}, 0.1 \mathrm{mM} \mathrm{MnCl}_{2}$, in PBS ( $\mathrm{pH}$ 6.8), for two 15-min washes and subsequently incubated with biotinylated isolectin-B4 (L-2140, Sigma-Aldrich, Sweden). A streptavidine-coupled Alexa-488 (Life Technologies) was used for visualization. Hindbrains were flat-mounted and imaged in a confocal (LSM700). The image analysis was done using CellProfiler (www.cellprofiler.org) with a pipeline as follows: an initial segmentation of the vessels was done using a fixed global threshold, followed by filtering out small particles. Next, a skeleton was computed from the binary vessel mask. From the skeleton, the number of branch points and the branch lengths were measured. Branches touching the border or very short branches were removed. For each branch, the mean distance values for the corresponding skeleton segment were calculated.

Transmission electron microscopy. Two sex- and age-matched mice (7-week-old male and female Vegfr2 $2^{\text {Y949F/Y949F }}$ and WT littermates) were perfusion fixed and their organs were collected and embedded in epoxy resin ${ }^{16}$. Briefly, mice were anaesthetized using a mixture of ketamine/xylazine and perfused via the left heart ventricle with oxygenated DMEM/ $1 \mathrm{mM} \mathrm{CaCl} / 5 \mathrm{mM}$ D-glucose. This was immediately followed by perfusion with $3 \%$ PFA/1.5\% glutaraldehyde $/ 5 \%$ sucrose in $0.1 \mathrm{M}$ phosphate buffer, $\mathrm{pH}$ 7.2. Organs (kidney and pancreas) were removed, cut in small blocks and further fixed ( $1 \mathrm{~h}$ at room temperature) in $2 \%$ glutaraldehyde in $0.1 \mathrm{M}$ sodium cacodylate buffer, rinsed in $0.1 \mathrm{M}$ cacodylate, postfixed ( $1 \mathrm{~h}$, on ice, in the dark) in $1 \% \mathrm{OsO} 4$, stained (over night, room temperature, in the dark) with $2 \%$ uranyl acetate, dehydrated in graded ethanol and LX-112 embedded (Ladd Research, Burlington, VT). Ultrathin sections ( $\sim 30 \mathrm{~nm})$ were obtained using an oscillating diamond knife (Diatome, Emsdiasum), further stained with $2 \%$ uranyl acetate followed by lead citrate, examined and imaged with a Jeol 1010 electron microscope using a bottom-mount AMD camera. Images were exported in TIFF format, and labelled and assembled using Adobe Photoshop and 
Illustrator CS6 (Adobe Systems). In some images, the contrast and brightness were post-processed in Adobe Photoshop using the respective linear functions.

RNA extraction and sequencing. RNA was extracted using the Qiagen RNeasy kit following the manufacturer's instructions and using lungs from 5- to 8-week-old mice. Extracted RNA quality was assessed with a BioAnalyzer (Agilent Technologies, Wilmington, DE, USA) and concentration estimated using the Qubit RNA BR Assay Kit (Thermo Ficher Scientific, Eugene, Oregon, USA). From $11 \mu \mathrm{g}$ of total RNA, mRNA purification was performed using Dynabeads mRNA Purification Kit (Ambion, Life Technologies AS, Oslo). mRNA libraries, three biological replicates per sample, were prepared using the Ion Total RNA-Seq Kit v2 (Life Technologies) using the standard protocol, and sequencing was performed on Ion Proton sequencer (Life Technologies). Libraries were barcoded three per PI v2 chip to achieve 20 million reads per replicate. Reads were processed, mapped to a reference genome $(\mathrm{mm} 10)$ and summarized using Torrent Suite version 5.0.2 and default settings. The statistical analysis of gene expression was performed in $\mathrm{R}$ using DESeq2 package (doi:10.1186/gb-2010-11-10-r106) with dispersion fit type set to local. The FPKM (fragments per kilobase per million) function from DESeq2 package was used to normalize reads per gene as RPKM (reads per kilobase per million), using the robust library size estimate. The RPKM values for selected genes were plotted on the $\log 10$ scale based on the folds of our genes of interest established in $\log 2$ fold change $(\log 2 \mathrm{FC}=1$ means the gene is upregulated twofolds).

\section{References}

1. Baluk, P., Hashizume, H. \& McDonald, D. M. Cellular abnormalities of blood vessels as targets in cancer. Curr. Opin. Genet. Dev. 15, 102-111 (2005).

2. Liao, D. \& Johnson, R. S. Hypoxia: a key regulator of angiogenesis in cancer. Cancer Metastasis Rev. 26, 281-290 (2007).

3. Dejana, E., Orsenigo, F. \& Lampugnani, M. G. The role of adherens junctions and VE-cadherin in the control of vascular permeability. J. Cell Sci. 121, 2115-2122 (2008).

4. Goel, S. et al. Normalization of the vasculature for treatment of cancer and other diseases. Physiol. Rev. 91, 1071-1121 (2011).

5. Ebos, J. M., Lee, C. R. \& Kerbel, R. S. Tumor and host-mediated pathways of resistance and disease progression in response to antiangiogenic therapy. Clin. Cancer Res. 15, 5020-5025 (2009).

6. Koch, S., Tugues, S., Li, X., Gualandi, L. \& Claesson-Welsh, L. Signal transduction by vascular endothelial growth factor receptors. Biochem. J. 437, 169-183 (2011).

7. Matsumoto, T. et al. VEGF receptor-2 Y951 signaling and a role for the adapter molecule TSAd in tumor angiogenesis. EMBO J. 24, 2342-2353 (2005).

8. Sun, Z. et al. VEGFR2 induces c-Src signaling and vascular permeability in vivo via the adaptor protein TSAd. J. Exp. Med. 209, 1363-1377 (2012).

9. Takahashi, T., Yamaguchi, S., Chida, K. \& Shibuya, M. A single autophosphorylation site on KDR/Flk-1 is essential for VEGF-A-dependent activation of PLC-gamma and DNA synthesis in vascular endothelial cells. EMBO J. 20, 2768-2778 (2001).

10. Sakurai, Y., Ohgimoto, K., Kataoka, Y., Yoshida, N. \& Shibuya, M. Essential role of Flk-1 (VEGF receptor 2) tyrosine residue 1173 in vasculogenesis in mice. Proc. Natl Acad. Sci. USA 102, 1076-1081 (2005).

11. Valenzuela, D. M. et al. High-throughput engineering of the mouse genome coupled with high-resolution expression analysis. Nat. Biotechnol. 21, 652-659 (2003).

12. Weis, S. et al. Src blockade stabilizes a Flk/cadherin complex, reducing edema and tissue injury following myocardial infarction. J. Clin. Invest. 113, 885-894 (2004).

13. Dejana, E. \& Vestweber, D. The role of VE-cadherin in vascular morphogenesis and permeability control. Prog. Mol. Biol. Transl. Sci. 116, 119-144 (2013).

14. Gerhardt, H. et al. VEGF guides angiogenic sprouting utilizing endothelial tip cell filopodia. J. Cell Biol. 161, 1163-1177 (2003).

15. Bentley, K. et al. The role of differential VE-cadherin dynamics in cell rearrangement during angiogenesis. Nat. Cell. Biol. 16, 309-321 (2014).

16. Stan, R. V. et al. The diaphragms of fenestrated endothelia: gatekeepers of vascular permeability and blood composition. Dev. Cell 23, 1203-1218 (2012).

17. Paolinelli, R., Corada, M., Orsenigo, F. \& Dejana, E. The molecular basis of the blood brain barrier differentiation and maintenance. Is it still a mystery? Pharmacol. Res. 63, 165-171 (2011).

18. Maes, W. \& Van Gool, S. W. Experimental immunotherapy for malignant glioma: lessons from two decades of research in the GL261 model. Cancer Immunol. Immunother. 60, 153-160 (2011).

19. Armulik, A. et al. Pericytes regulate the blood-brain barrier. Nature 468, 557-561 (2010).

20. Hanahan, D. Heritable formation of pancreatic beta-cell tumours in transgenic mice expressing recombinant insulin/simian virus 40 oncogenes. Nature 315, 115-122 (1985)

21. Anderberg, C. et al. Deficiency for endoglin in tumor vasculature weakens the endothelial barrier to metastatic dissemination. J. Exp. Med. 210, 563-579 (2013).
22. Overwijk, W. W. \& Restifo, N. P. B16 as a mouse model for human melanoma. Curr. Protoc. Immunol. Chapter 20, Unit 20.1 (2001).

23. Graham, M. M. \& Evans, M. L. A simple, dual tracer method for the measurement of transvascular flux of albumin into the lung. Microvasc. Res. $\mathbf{4 2}$, 266-279 (1991).

24. Hayashi, M. et al. VE-PTP regulates VEGFR2 activity in stalk cells to establish endothelial cell polarity and lumen formation. Nat. Commun. 4, 1672 (2013).

25. Eliceiri, B. P. et al. Selective requirement for Src kinases during VEGF-induced angiogenesis and vascular permeability. Mol. Cell 4, 915-924 (1999).

26. Di Lorenzo, A., Fernandez-Hernando, C., Cirino, G. \& Sessa, W. C. Akt1 is critical for acute inflammation and histamine-mediated vascular leakage. Proc. Natl Acad. Sci. USA 106, 14552-14557 (2009).

27. Orsenigo, F. et al. Phosphorylation of VE-cadherin is modulated by haemodynamic forces and contributes to the regulation of vascular permeability in vivo. Nat. Commun. 3, 1208 (2012).

28. Koch, S. et al. NRP1 presented in trans to the endothelium arrests VEGFR2 endocytosis, preventing angiogenic signaling and tumor initiation. Dev. Cell 28, 633-646 (2014)

29. Kohn, S., Nagy, J. A., Dvorak, H. F. \& Dvorak, A. M. Pathways of macromolecular tracer transport across venules and small veins. Structural basis for the hyperpermeability of tumor blood vessels. Lab. Invest. 67, 596-607 (1992).

30. Nagy, J. A., Benjamin, L., Zeng, H., Dvorak, A. M. \& Dvorak, H. F. Vascular permeability, vascular hyperpermeability and angiogenesis. Angiogenesis 11, 109-119 (2008).

31. McDonald, D. M., Thurston, G. \& Baluk, P. Endothelial gaps as sites for plasma leakage in inflammation. Microcirculation 6, 7-22 (1999).

32. Berse, B., Brown, L. F., Van de Water, L., Dvorak, H. F. \& Senger, D. R. Vascular permeability factor (vascular endothelial growth factor) gene is expressed differentially in normal tissues, macrophages, and tumors. Mol. Biol. Cell 3, 211-220 (1992).

33. Chen, X. L. et al. VEGF-induced vascular permeability is mediated by FAK. Dev. Cell 22, 146-157 (2012).

34. Jean, C. et al. Inhibition of endothelial FAK activity prevents tumor metastasis by enhancing barrier function. J. Cell Biol. 204, 247-263 (2014).

35. Schleicher, M. et al. The Akt1-eNOS axis illustrates the specificity of kinase-substrate relationships in vivo. Sci. Signal. 2, ra41 (2009).

36. Forstermann, U. \& Sessa, W. C. Nitric oxide synthases: regulation and function. Eur. Heart J. 33, 829-837 837a-837d (2012).

37. Gratton, J. P. et al. Selective inhibition of tumor microvascular permeability by cavtratin blocks tumor progression in mice. Cancer Cell 4, 31-39 (2003).

38. Manni, S., Kisko, K., Schleier, T., Missimer, J. \& Ballmer-Hofer, K. Functional and structural characterization of the kinase insert and the carboxy terminal domain in VEGF receptor 2 activation. FASEB J. 28, 4914-4923 (2014).

39. Kuppers, V., Vockel, M., Nottebaum, A. F. \& Vestweber, D. Phosphatases and kinases as regulators of the endothelial barrier function. Cell Tissue Res. 355, 577-586 (2014)

40. Bergers, G. \& Hanahan, D. Modes of resistance to anti-angiogenic therapy. Nat. Rev. Cancer 8, 592-603 (2008)

41. Butler, T. P., Grantham, F. H. \& Gullino, P. M. Bulk transfer of fluid in the interstitial compartment of mammary tumors. Cancer Res. 35, 3084-3088 (1975).

42. Gullino, P. M. \& Grantham, F. H. The vascular space of growing tumors. Cancer Res. 24, 1727-1732 (1964).

43. Schulte, D. et al. Stabilizing the VE-cadherin-catenin complex blocks leukocyte extravasation and vascular permeability. EMBO J. 30, 4157-4170 (2011).

44. Mohanty, S. \& Xu, L. Experimental metastasis assay. J. Vis. Exp. 42, 1942-1944 (2010).

45. Benest, A. V. et al. Angiopoietin-2 is critical for cytokine-induced vascular leakage. PLoS ONE 8, e70459 (2013).

46. Carlsson, P. O., Andersson, A. \& Jansson, L. Pancreatic islet blood flow in normal and obese-hyperglycemic (ob/ob) mice. Am. J. Physiol. 271, E990-E995 (1996).

47. Jansson, L. \& Hellerstrom, C. A rapid method of visualizing the pancreatic islets for studies of islet capillary blood flow using non-radioactive microspheres. Acta Physiol. Scand. 113, 371-374 (1981).

48. Lau, J. et al. Implantation site-dependent dysfunction of transplanted pancreatic islets. Diabetes 56, 1544-1550 (2007).

\section{Acknowledgements}

This work was supported by grants from the Swedish Cancer Society, the Swedish Science Council, the Knut and Alice Wallenberg Foundation and the Association for International Cancer Research/Worldwide Cancer Research (grant 13-1295), to L.C.-W., from the Swiss National Science Foundation (grant 31003A-130463, the Oncosuisse (grant OC201200-08 -2007) to K.B.-H., from the European Research Council (294556 BBBARRIER) and Leducq Foundation (Sphingonet) to C.B., and from the National Institute of Health (CA172983 and CA175592) to R.V.S. E.G. was supported by a Wenner-Gren Foundation postdoctoral scholarship. We acknowledge expert assistance by the following SciLifeLab facilities: NGI; BioVis imaging facility; BILS; and UPPMAX SNIC. We also thank Petter Ranefall, Center for Image Analysis, SciLifeLab, Uppsala 
University, for expert assistance with image rendering and analysis. We thank Dr Doug Hanahan, Swiss Institute for Experimental Cancer Research, for antibodies against SV40 T, Dr Géza Sáfrány, Frédéric Joliot-Curie National Research Institute for Radiobiology and Radiohygiene, Budapest, Hungary, for sharing the GL261 cells and Dr David D. Schlaepfer, University of California at San Diego for dsRed-B16F10 cells.

\section{Author contributions}

X.L., N.P., E.O.S., N.H., F.R., M.S.-J., E.G., K.B., C.T., R.V.S., V.T., L.J. and L.C.-W. planned and executed experiments; all authors analysed and interpreted data, and assisted in putting the manuscript together.

\section{Additional information}

Supplementary Information accompanies this paper at http://www.nature.com/ naturecommunications
Competing financial interests: The authors declare no competing financial interests.

Reprints and permission information is available online at http://npg.nature.com/ reprintsandpermissions/

How to cite this article: Li, X. et al. VEGFR2 pY949 signalling regulates adherens junction integrity and metastatic spread. Nat. Commun. 7:11017 doi: 10.1038/ncomms11017 (2016).

(c) (i) This work is licensed under a Creative Commons Attribution 4.0 International License. The images or other third party material in this article are included in the article's Creative Commons license, unless indicated otherwise in the credit line; if the material is not included under the Creative Commons license, users will need to obtain permission from the license holder to reproduce the material. To view a copy of this license, visit http://creativecommons.org/licenses/by/4.0/ 